\title{
Mechanisms Underlying the Cardioprotection of YangXinDingJi Capsule against Myocardial Ischemia in Rats
}

\author{
Miaomiao Liu, ${ }^{1}$ Yurun Xue, ${ }^{1}$ Yingran Liang, ${ }^{1}$ Yucong Xue, ${ }^{1}$ Xue Han, ${ }^{1,2}$ Ziliang Li, ${ }^{3}$ \\ and Li Chu $\mathbb{D i D}^{1,4}$ \\ ${ }^{1}$ School of Pharmacy, Hebei University of Chinese Medicine, Shijiazhuang 050200, Hebei, China \\ ${ }^{2}$ Hebei Higher Education Institute Applied Technology Research Center on TCM Formula Preparation, \\ Shijiazhuang 050091, China \\ ${ }^{3}$ School of Basic Medicine, Hebei University of Chinese Medicine, Shijiazhuang 050200, Hebei, China \\ ${ }^{4}$ Hebei Key Laboratory of Integrative Medicine on Liver-Kidney Patterns, Shijiazhuang 050200, Hebei, China \\ Correspondence should be addressed to Li Chu; chuli0614@126.com
}

Received 30 July 2020; Revised 26 August 2020; Accepted 20 October 2020; Published 17 November 2020

Academic Editor: Hong Chang

Copyright (c) 2020 Miaomiao Liu et al. This is an open access article distributed under the Creative Commons Attribution License, which permits unrestricted use, distribution, and reproduction in any medium, provided the original work is properly cited.

\begin{abstract}
Background. YangXinDingJi (YXDJ) capsule is one of traditional Chinese medicines (TCMs) derived from Zhigancao decoction, which is usually used for the treatment of cardiovascular disease in China. Aim of the Study. Cardiovascular events are one of the leading causes of death worldwide. Myocardial ischemia (MI) severely reduces myocyte longevity and function. The YangXinDingJi (YXDJ) capsule has been used in the treatment of clinical cardiac disease in China. Nevertheless, the underlying cellular mechanisms for the benefits to heart function resulting from the use of this capsule are still unclear. The aim of this study was to evaluate the protective effects of the YXDJ on isoprenaline-induced MI in rats and to clarify its underlying myocardial protective mechanisms based on L-type calcium channels and myocardial contractility. Materials and Methods. Rats were randomly divided into five groups with ten rats in each group: (1) control; (2) ISO-induced model; (3) high-dose YXDJ (2.8 g/kg/day intraperitoneally for five days), (4) low-dose YXDJ (1.4 g/kg/day for five days); and (5) verapamil ( $n=10$ in each group). Isoproterenol (ISO) was injected subcutaneously for two consecutive days to induce the rat model of MI. Heart and biochemical parameters were obtained. The patch-clamp technique was used to observe the regulatory effects of YXDJ on the L-type calcium current $\left(\mathrm{I}_{\mathrm{Ca}-\mathrm{L}}\right)$ in isolated cardiomyocytes. An IonOptix MyoCam detection system was used to observe the contractility of YXDJ on isolated cardiomyocytes. Results. YXDJ caused a significant improvement in pathological heart morphology and alleviated oxidative stress and inflammatory responses. Exposure to YXDJ caused a decrease in blockade of $\mathrm{I}_{\mathrm{Ca}-\mathrm{L}}$ in a concentration-dependent manner. Conclusions. The results indicate that YXDJ significantly inhibited inflammatory cytokine expressions, oxidative stress, and L-type $\mathrm{Ca}^{2+}$ channels, and decreased contractility in isolated rat cardiomyocytes. These findings may be relevant to the cardioprotective efficacy of YXDJ.
\end{abstract}

\section{Introduction}

As a fatal disease, ischemic heart disease (IHD) has become one of the most serious health problems both in developing and developed countries [1, 2]. Prolonged ischemia can lead to reperfusion injury to cardiac myocytes, which may include varying degrees of cell death and myocardial edema [3]. Several factors are known to increase the risk for myocardial ischemia (MI); for example, beta-adrenergic stimulation is thought to exacerbate ongoing myocardial infarction [4]. Isoprenaline (ISO), a nonselective $\beta$-adrenoceptor agonist, is widely used to induce MI in experimental rat models of cardiac disease. In these models, activation of the adrenergic system causes severe stress in the myocardium by inducing an increase in the L-type $\mathrm{Ca}^{2+}$ channel (LTCC) activity [5].

In the occurrence and maintenance of MI, a number of mechanisms are involved that cause an imbalance of 
intracellular $\mathrm{Ca}^{2+}$. LTCCs are the main routes for calcium entry into cardiac myocytes, which is responsible for initiating contraction in the heart $[6,7]$. LTCCs (also known as voltage-gated calcium channels, $\mathrm{Cav}_{1.2}$ channels, and dihydropyridine receptors) can respond to small changes in membrane potential [8]. This allows LTCC to create a "calcium window" that provides a small, sustained influx of $\mathrm{Ca}^{2+}$ into the cell that can activate the contractile mechanism [9]. Membrane depolarization via the action potential leads to the opening of L-type channels, which leads to heart contractions during systole [10]. Plasma membrane influx of $\mathrm{Ca}^{2+}$ by LTCCs leads to $\mathrm{Ca}^{2+}$-induced $\mathrm{Ca}^{2+}$ release, which in turn regulates cardiac contractility. Oxidative stress can cause some cellular defects such as decreasing of the sarcolemmal Ca ${ }^{2+}$ ATPase pump and $\mathrm{Na}^{+}-\mathrm{K}^{+}$ATPase activities. These alterations lead to a decrease in the $\mathrm{Ca}^{2+}$ effluxes and an increase in the $\mathrm{Ca}^{2+}$ influxes, respectively [11].

Studies on the association of $\mathrm{Ca}^{2+}$ increase with an increase in oxidative stress have been contradictory, and the sequence of events remains controversial. The increase in cytosolic $\mathrm{Ca}^{2+}$ concentration is induced by reactive oxygen species (ROS) [12]. An increase in $\mathrm{Ca}^{2+}$ is a constant feature of pathological states associated with oxidative stress [13]. It has been found that myocardial ischemia is related to inflammatory response and oxidative stress $[14,15]$. Oxidative stress is an imbalance between ROS and antioxidants and is actually the imbalance between the generations of ROS and body antioxidant defense systems that associated with many noncommunicable diseases, such as cancer, heart disease, and diabetes [16]. Excessive ROS causes oxidative damage to important cellular components such as DNA, protein, and lipid membranes. In MI, hypoxia and reoxygenation occur, which may induce the excessive production of ROS in cardiac tissues [17]. Free radicals are typically scavenged by antioxidant enzymes including superoxide dismutase (SOD) and malondialdehyde (MDA), and previous studies have suggested that the antioxidant activities of these enzymes may be reduced in patients following MI or in those with ischemic heart disease $[18,19]$. After an MI, inflammatory response occurs, and macrophage infiltration gradually increases. In addition, resent research suggests that MI injury is a result of an increased expression of inflammatory cytokines [20]. Expression and release of inflammatory cytokines such as interleukin (IL)-6, tumor necrosis factor (TNF)- $\alpha$, and several chemokines contribute to upregulation of cell-adhesion molecules, cardiac functional depression, and apoptosis [21].

Traditional Chinese medicine (TCM) has been widely used in clinical treatment of heart disease in China [22]. Zhigancao decoction is a TCM that originated from "shang han lun" written by Zhang Zhongjing in the Eastern Han Dynasty [23]. The therapeutic effects of Zhigancao decoction medicated serum are comparable to routinely used antiarrhythmic medicines. Long-term use in patients has also demonstrated good tolerance to the drug in patients [24]. YangXinDingJi capsule (YXDJ) evolved from the Zhigancao decoction, which is composed of Radix ophiopogonis, Rehmannia, licorice, ginger, red ginseng, jujube, donkey hide gelatin, black sesame, and cinnamon twig (see Table 1).
This prescription was approved by the China Food and Drug Administration (license no. Z19991082) and is used in the treatment of palpitations caused by heart syndrome. According to previous research, the main constituents in YXDJ capsule, including liquiritin, glycyrrhizic acid, cinnamic acid, and cinnamic aldehyde, were analyzed by the HPLC analysis method using a Diamonsil C18 column and the results showed good linear relationships. The ranges of liquiritin, glycyrrhizic acid, cinnamic acid, and cinnamic aldehyde were $1.00-80.24 \mu \mathrm{g} / \mathrm{mL}(r=0.9990), 2.52-100.70$ $\mu \mathrm{g} / \mathrm{mL} \quad(r=0.9997), \quad 0.50-40.40 \mu \mathrm{g} / \mathrm{mL} \quad(r=1.0000)$, and $0.66-52.96 \mu \mathrm{g} / \mathrm{mL} \quad(r=1.0000)$, respectively [25]. A recent clinical trial confirmed its effectiveness for improving symptoms and reducing angina pectoris and complications along with few adverse effects [26]. However, the precise mechanism underlying the cellular $\mathrm{Ca}^{2+}$ homeostasis of YXDJ protects against cardiac remains poorly understood. We hypothesized that YXDJ plays a role in myocardial protection by regulating $\mathrm{Ca}^{2+}$ homeostasis, reducing oxidative stress, suppressing inflammatory cytokine expressions, and reducing cytoplasmic myocardial concentration.

In this paper, we evaluated the LTCC current $\left(\mathrm{I}_{\mathrm{Ca}-\mathrm{L}}\right)$ changes with respect to relieving oxidative stress of YXDJ in cardiomyocytes. Further study on the cellular mechanisms of YXDJ will not only contribute to a better understanding of the efficacies in clinical treatments but also provide experimental evidence for rational applications.

\section{Materials and Methods}

2.1. Reagents. YangXinDingJi capsule (batch no. 06720021241, $0.5 \mathrm{~g} /$ capsule, approval number: Z19991082) was acquired from Yongfeng Pharmaceutical Co., Ltd. (Shijiazhuang, China). ISO was acquired from Amylet Scientific Inc. (Michigan, USA). Verapamil was acquired from Hefeng Pharmaceutical Co., Ltd. (Shanghai, China). Unless otherwise stated, other chemical reagents were obtained from Sigma-Aldrich (St. Louis, MO, USA). All solvents used were of analytical grade.

2.2. Animals. A total of 100 adult male Sprague-Dawley rats weighing $200 \pm 20 \mathrm{~g}$ are used in this study. The rats were provided by the Experimental Animal Center of Hebei Province. Thirty-eight rats were used for patch clamp to record $\mathrm{Ca}^{2+}$ currents, twelve rats were used to detect cell shortening, and fifty rats were used for experiment in vivo. Male adult SD rats $(200 \pm 20 \mathrm{~g})$ were exposed to an environment of $22 \pm 2^{\circ} \mathrm{C}$, and humidity was $55 \pm 5 \%$ with a $12 \mathrm{~h}$ light- $12 \mathrm{~h}$ dark cycle and adequate food and water supplies. All studies were performed in conformity with the guidelines for care and standard experimental animals' study ethical protocols and approved by the Ethics Committee for Animal Experiments of Hebei University of Chinese Medicine.

2.3. Induction of Myocardial Ischemia Injury. After acclimatization to laboratory conditions, fifty male SD rats were randomly assigned into five experimental groups $(n=10$ per group): (1) control group (CON); (2) ISO-induction group 
TABLe 1: Description of YangXinDingJi capsule.

\begin{tabular}{|c|c|c|c|c|c|c|}
\hline Chinese name & Latin name & Family & Scientific name & Plant part & $\%$ & $\begin{array}{l}\text { Origin } \\
\text { (China) }\end{array}$ \\
\hline Dihuang & Radix et Rhizoma Rhei & Polygonaceae & Rehmannia glutinosa (Gaertn.) DC. & Rhizome & 37 & Si Chuang \\
\hline Dazao & Fructus Jujubae & Rhamnaceae & Ziziphus jujuba Mill. & Fruit & 14 & Hebei \\
\hline Maidong & Ophiopogonis Radix & Liliaceae & $\begin{array}{l}\text { Ophiopogon japonicus (Thunb.) Ker } \\
\text { Gawl. }\end{array}$ & Root & 13 & Guang Dong \\
\hline Zhigancao & $\begin{array}{l}\text { Radix Glycyrrhizae } \\
\text { Preparata }\end{array}$ & Leguminosae & Glycyrrhiza glabra L. & Rhizome & 9 & Gan Su \\
\hline Heizhima & Sesami Semen Nigrum & Pedaliaceae & Sesamum indicum L. & Seed & 7 & Hu Bei \\
\hline Guizhi & Ramulus Cinnamomi & Lauraceae & Cinnamomum cassia (L.) J. Presl & Branch & 6 & Guang Dong \\
\hline Shengjiang & Rhizoma Zingiberis Recens & Zingiberaceae & Zingiber officinale Roscoe & Rhizome & 6 & Hebei \\
\hline Ejiao & Asini Corii Colla & Equidae & Equus asinus L. & Hide gelatin & 4 & Shan Dong \\
\hline Hongshen & Radix Ginseng Rubra & Araliaceae & Panax ginseng CA Mey. & Rhizome & 4 & Ji Lin \\
\hline
\end{tabular}

(ISO); (3) verapamil treatment group (VER); (4) high-dose YXDJ $\left(\mathrm{YXDJ}_{\mathrm{H}}\right)$; and (5) low-dose YXDJ $\left(\mathrm{YXDJ}_{\mathrm{L}}\right)$. The CON and ISO groups were gavaged with distilled water. $\mathrm{YXDJ}_{\mathrm{H}}$ and $\mathrm{YXDJ}_{\mathrm{L}}$ groups were gavaged with YXDJ (2.8 and $1.4 \mathrm{~g}$ / $\mathrm{kg} /$ day, respectively). The VER group was intraperitoneally injected with verapamil $(2 \mathrm{mg} / \mathrm{kg} /$ day $)$. After seven days of continuous administration, rats were subcutaneously injected with ISO for two consecutive days $(85 \mathrm{mg} / \mathrm{kg} /$ day, except in the CON group). At the end of the experiment, the rats were anesthetized with sodium pentobarbital $(40 \mathrm{mg} / \mathrm{kg})$ and hearts were removed and examined as described below.

2.4. Electrocardiogram. At the end of the experimental period, the anesthetized rats were examined by electrocardiogram (ECG). The needle electrodes were linked to the rat's right arm, front arms, and left leg. ECGs were recorded with needle electrodes and a BL-420S Biological Data Acquisition \& Analysis System (Chengdu TEM Technology Co., Ltd., China).

2.5. Myocardium Histopathology. The animal hearts were dissected rapidly and fixed in $4 \%$ paraformaldehyde. The heart tissues obtained from rats were embedded in paraffin and sectioned into at $4-\mu \mathrm{m}$ slices. The heart tissues were processed for sectioning and staining by standard histological methods. The results were observed under a microscope (Leica DM4000B, Solms, Germany).

2.6. Ultrastructural Examination by Transmission Electron Microscopy. Rats were anesthetized, and the hearts were quickly removed. Heart tissues were cut into small pieces (approximately, $1 \mathrm{~mm}^{3}$ ). These pieces were then rapidly fixed with $2.5 \%$ glutaraldehyde in $0.1 \mathrm{~mol} / \mathrm{L}$ sodium phosphate buffer ( $\mathrm{pH} 7.4$ ) at $4^{\circ} \mathrm{C}$ for $3 \mathrm{~h}$ and then fixed again with $1 \%$ osmium tetroxide at $4^{\circ} \mathrm{C}$ for $1 \mathrm{~h}$. After dehydration with a graded ethanol series, the thin sections were cut with a Leica EM UC6 (Leica Co, Austria) ultramicrotome. A sample was embedded in epon812, and the section was viewed and photographed on a Hitachi 7650 transmission electron microscope (TEM) from (Hitachi, Japan) at $80 \mathrm{kV}$.
2.7. Serum Preparation for Detection of $C K, L D H, S O D$, and $M D A$. Blood was obtained from rats that had been anesthetized at the end of 7 days of treatment. Serum was pipetted and saved at $-20^{\circ} \mathrm{C}$ following centrifugation at $3000 \mathrm{rpm}$ at $4^{\circ} \mathrm{C}$ for $10-15 \mathrm{~min}$. CK and LDH, SOD activity, and MDA levels were determined by standard commercial kits (Jiancheng, Nanjing, China) according to the manufacturer's instructions.

2.8. Detection of Intracellular ROS. Dihydroethidium (DHE) oxidation is commonly used as a method for monitoring cellular production of ROS. The fluorescence of DHE probe was used to evaluate ROS production in heart tissues. We studied quantitative changes in DHE oxidation products. ROS generation was labeled with the red fluorescence, and visualized and analyzed using a high-content screening system. All experiments were repeated at least three times.

2.9. Inflammation Assay. After centrifugation, serum was collected. IL- 6 and TNF- $\alpha$ levels in serum were determined to examine the relationship between the cardioprotective effects of YXDJ and inflammatory cytokine levels. Serum level of IL-6 was calculated from the kit standards and expressed as $\mathrm{pg} / \mathrm{mL}$, and TNF- $\alpha$ was expressed as $\mathrm{pg} / \mathrm{mL}$.

2.10. Detection of Calcium Concentration in Myocardial Tissues. The $\mathrm{Ca}^{2+}$ concentration was determined in heart tissue. Myocardial tissues were placed in a buffered solution $(\mathrm{m} / \mathrm{V}=1: 9)$. The homogenate was centrifuged at $1000 \mathrm{rpm}$ at $4^{\circ} \mathrm{C}$ for $10 \mathrm{~min}$ to remove cell debris, and the supernatant was saved for all downstream biochemical analyses. $\mathrm{Ca}^{2+}$ concentrations were evaluated by Coomassie Brilliant Blue staining with a commercial kit (Jiancheng, Nanjing, China).

2.11. Isolation of Cardiomyocytes. Single myocardial cells were obtained via enzymolysis. Rats were intraperitoneally injected with $500 \mathrm{IU} / \mathrm{kg}$ heparin and anesthetized with $40 \mathrm{mg} / \mathrm{kg}$ sodium pentobarbital. The heart was quickly removed from the rat, placed on a Langendorff instrument for perfusion with an oxygenated free calcium Tyrode's solution, which contained (mM) $\mathrm{NaH}_{2} \mathrm{PO}_{4} 0.33, \mathrm{NaCl} 135, \mathrm{KCl}$ 
5.4, glucose 10, $\mathrm{MgCl}_{2} 1.0$, and HEPES 10 (pH adjusted to 7.4 with $3 \mathrm{M} \mathrm{NaOH}$ ). After clearance of the blood, the enzyme solution containing $4 \mathrm{~g} / \mathrm{L}$ taurine, $10 \mathrm{~g} / \mathrm{L}$ bovine serum albumin (Roche, Basel, Switzerland), and $4 \mathrm{~g} / \mathrm{L}$ collagenase type II (GIBCO, Invitrogen, Carlsbad, CA, USA) was perfused for 15 to $25 \mathrm{~min}$ at $37^{\circ} \mathrm{C}$ until the heart was soft. After perfusion, the heart was placed into Krebs buffer (KB) and the heart tissue was dissected into small pieces. The cardiomyocytes were preserved in $\mathrm{KB}$ solution (bubbled with $\mathrm{O}_{2}$ ) at a temperature of $23-25^{\circ} \mathrm{C}$ for up to 1 to $2 \mathrm{~h}$ before the experiment.

2.12. Electrophysiological Techniques. Inward $\mathrm{Ca}^{2+}$ currents were recorded from rat myocytes. Whole-cell L-type $\mathrm{Ca}^{2+}$ currents were recorded using the conventional patch-clamp technique, as previously described [27]. Pipettes of 4 to $6 \mathrm{M} \Omega$ resistance were pulled from borosilicate glass (Kimax 51, KIMBLE Glass Inc.) Microelectrodes had resistances of 4 to $6 \mathrm{M} \Omega$ and were lightly fire polished and filled with an intracellular solution containing $\mathrm{MgCl}_{2} 2.0 \mathrm{mM}$, glucose $10 \mathrm{mM}, \mathrm{CaCl}_{2} 1.8 \mathrm{mM}$, TEA-Cl $140 \mathrm{mM}$, HEPES $10 \mathrm{mM}$, and TTX $10 \mu \mathrm{M}$ (pH 7.4). Seventy to ninety percent of the series resistance was compensated. Cells were bathed in a potassium-free extracellular solution that contained (in mM) $140 \mathrm{NaCl}, 5.4 \mathrm{CsCl}, 2.5 \mathrm{CaCl}_{2}, 0.5 \mathrm{MgCl}_{2}, 11$ glucose, and 5.5 HEPES ( $\mathrm{pH}$ 7.4). YXDJ capsule was dissolved in distilled water before, and then dissolved in extracellular solution when patch clamp is used to recorded $\mathrm{Ca}^{2+}$ currents at the following concentration of $100-500 \mu \mathrm{g} / \mathrm{mL}$. Wholecell patch-clamp experiments were carried out at room temperature $\left(20-25^{\circ} \mathrm{C}\right)$. For all experiments, the holding potential was $-80 \mathrm{mV}$ and currents were elicited by a $200-$ msec depolarizing pulse. Data were analyzed using the pCLAMP (Axon Instruments, Inc.).

2.13. Measurements of Cell Shortening. Ventricular myocytes were placed at the bottom of the glass chamber, and the normal Tyrode's solution flowed at a speed of $1 \mathrm{~mL} / \mathrm{min}$. Cell activity could be observed with an inverted microscope and a detection system placed on the glass chamber. Cell shortening at $500 \mu \mathrm{g} / \mathrm{mL}$ of YXDJ was measured to determine the effects of YXDJ on myocardial contractility. Myocytes with clear edges were selected to measure cell shortening before and after MI. The contractions of ventricular myocytes were assessed with an IonOptix MyoCam detection system (IonOptix, Milton, MA, USA).

2.14. Statistical Analysis. The steady-state activation and inactivation curves of $\mathrm{I}_{\mathrm{Ca}-\mathrm{L}}$ were fitted by Boltzmann functions. These conductances were normalized to their individual maximal conductance. All the values are represented as mean \pm standard error of the mean (SEM) and were analyzed by one-way analysis of variance (ANOVA). The statistical differences among different groups were analyzed by Student's $t$-test. $P$ values of $<0.05$ were considered significant.

\section{Result}

3.1. Effects of YXDJ on Electrocardiography. Analysis of electrocardiography patterns was done to determine the actions of YXDJ on ISO-induced MI animals. As shown in Figures $1(\mathrm{a})$ and 1(b), the obvious reduction in the J-point elevation and heart rate was achieved in the YXDJ groups at both the high and low doses compared to ISO $(P<0.05$; $P<0.01)$. Compared with CON, the heart rate and J-point of ISO-induced rats were evidently elevated.

Figure 1(c) shows sample ECG tracings from the experimental rats. The ECG of the CON group was normal. In the ISO group, the amplitude of the $\mathrm{R}$ wave decreased and the ST interval increased, suggesting that ISO induced a reduction in the amplitude of ECG waves and an increase in frequency. The YXDJ group and VER group showed a recovered $\mathrm{R}$ wave amplitude and ST interval.

\subsection{Effects of YXDJ on the Pathological Changes of Rat Hearts.} The biochemical alterations mentioned above could be correlated with the histological changes in the heart, as shown in Figure 2. According to the hematoxylin and eosin (H\&E) staining of the heart tissue, the heart tissue showed the normal histology of the CON group, which was composed of a natural muscle fibril structure. However, H\&E staining showed that ISO-induced structural alterations in the myocardium in which cardiomyocytes appeared smaller, cardiac muscle fiber was disorganized, and vestigial infiltrating inflammatory cells were present. The damage in the YXDJ and VER administration groups was lower than that in the ISO group.

\subsection{Effects of YXDJ on the Myocardial Mitochondrion} Ultrastructure. Mitochondrial ultrastructure was observed by TEM. As shown in Figure 3, the ultrastructure of the cardiac muscle from rats of the CON and VER groups was identical. However, myocardial mitochondria from the ISO group suffered substantial structural damage, including disordered mitochondrial distribution with disarranged and obscure crista and vacuoles within the matrix accompanying by disrupted sarcomere and myofilament. Noticeably, YXDJ pretreated alleviated these deleterious effects of mitochondrial ultrastructure induced by MI injury.

3.4. Effects of YXDJ on Cardiac Marker Enzymes. Cardiac markers in blood are the mainstay for cardiac damage diagnosis. As shown in Figure 4, a significant increase in cardiac markers was noted after ISO induction $(P<0.05)$ compared with the CON group. The serum CK and LDH activities of rats in the VER- and YXDJ-treated groups were significantly lower than those in the ISO group $(P<0.05$, $P<0.01)$.

3.5. Effects of YXDJ on SOD and MDA in Serum. To investigate the antioxidative effects of YXDJ, the action of YXDJ on ISO-induced SOD activity and MDA levels were detected. The activity of SOD in serum, as shown in Figure 5, ISO 


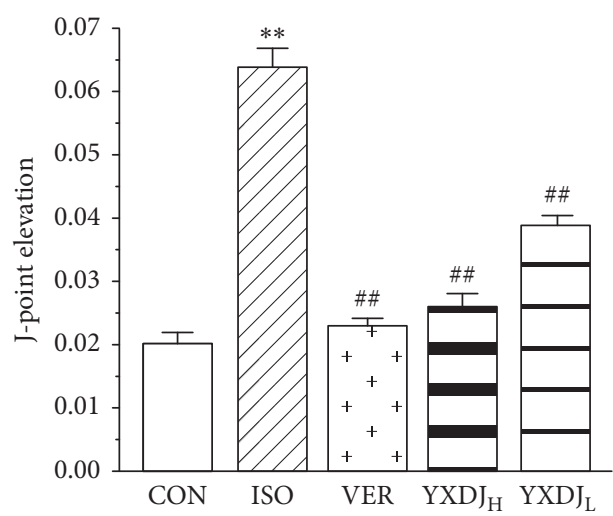

(a)
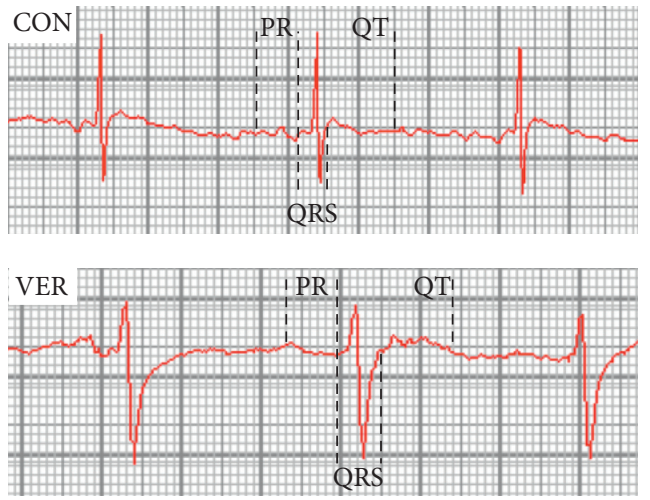

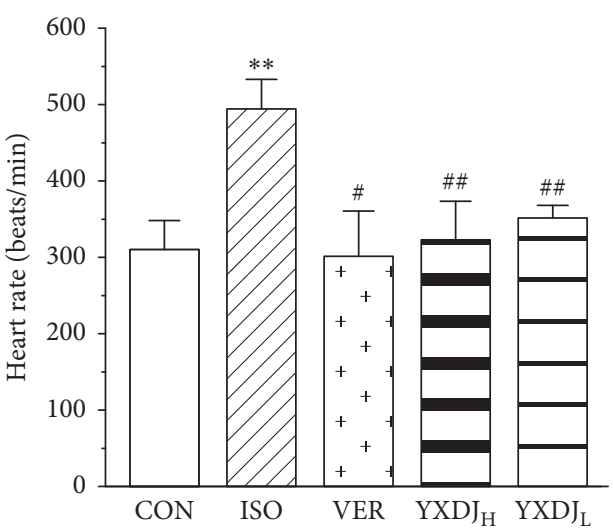

(b)
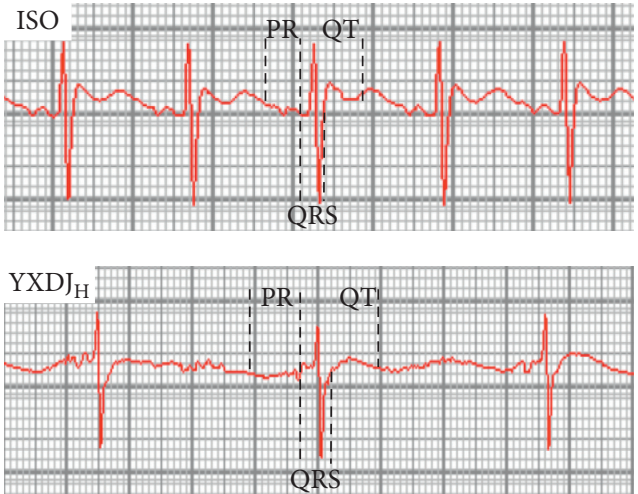

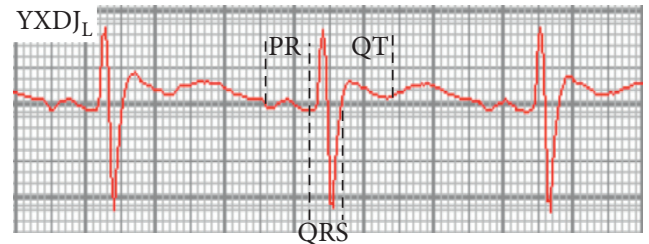

(c)

Figure 1: Actions of YangXinDingJi (YXDJ) on the electrocardiogram (ECG): (a) statistical analysis of J-point elevation, (b) heart rate, and (c) representative ECG tracings. Values represent the mean $\pm \mathrm{SE}$, for 10 rats in each group. ${ }^{* *} P<0.05$ versus control $(\mathrm{CON})$; ${ }^{\#} P<0.05$, \#\# $P<0.01$ versus isoproterenol (ISO).

group rats was markedly lower than that of the CON rats $(P<0.05)$. However, after treatment with YXDJ $(2.8$ and $1.4 \mathrm{~g} / \mathrm{kg} /$ day) and VER, the activity of SOD evidently increased $(P<0.05 ; P<0.01)$. MDA content was significantly higher in the ISO group than in the CON group $(P<0.05)$. Meanwhile, compared with the ISO-induced rats, the MDA levels significantly reduced after treatment with YXDJ (2.8 and $1.4 \mathrm{~g} / \mathrm{kg} /$ day $)$ and VER $(P<0.05)$.

3.6. Effects of YXDJ on ROS Release. The effect of YXDJ on the ISO-induced MI was evaluated with fluorescence of a dihydroethidium probe, specifically for assessing myocardial tissue ROS. As shown in Figure 6, VER obviously caused a reduction in the production of ROS. ROS evaluation revealed similar results in animals treated with YXDJ at both high and low does $(P<0.05 ; P<0.01)$. The results implied that YXDJ could cause a reduction in ISO-induced oxidative stress.
3.7. Effects of YXDJ on IL-6 and TNF- $\alpha$. To further evaluate and validate the protective function of YXDJ during MI injury, we measured the levels of IL- 6 and TNF- $\alpha$ in serum. The levels of TNF- $\alpha$ and IL- 6 were significantly increased after ISO induction. Preprocessing by YXDJ caused a decrease in the expressions of IL- 6 and TNF- $\alpha$ as shown in Figures 7(a) and 7(b) $(P<0.01)$. However, serum IL-6 and TNF- $\alpha$ concentrations in VER and high-dose YXDJ groups remained higher than those in the low-dose YXDJL group.

3.8. Effects of YXDJ on Calcium Concentration. As shown in Figure 8, ISO induction of MI resulted in a significant increase in the $\mathrm{Ca}^{2+}$ concentration of the heart tissue relative to the CON. However, compared with the ISO group, the YXDJ group and VER group were significantly decreased $(P<0.05)$. 

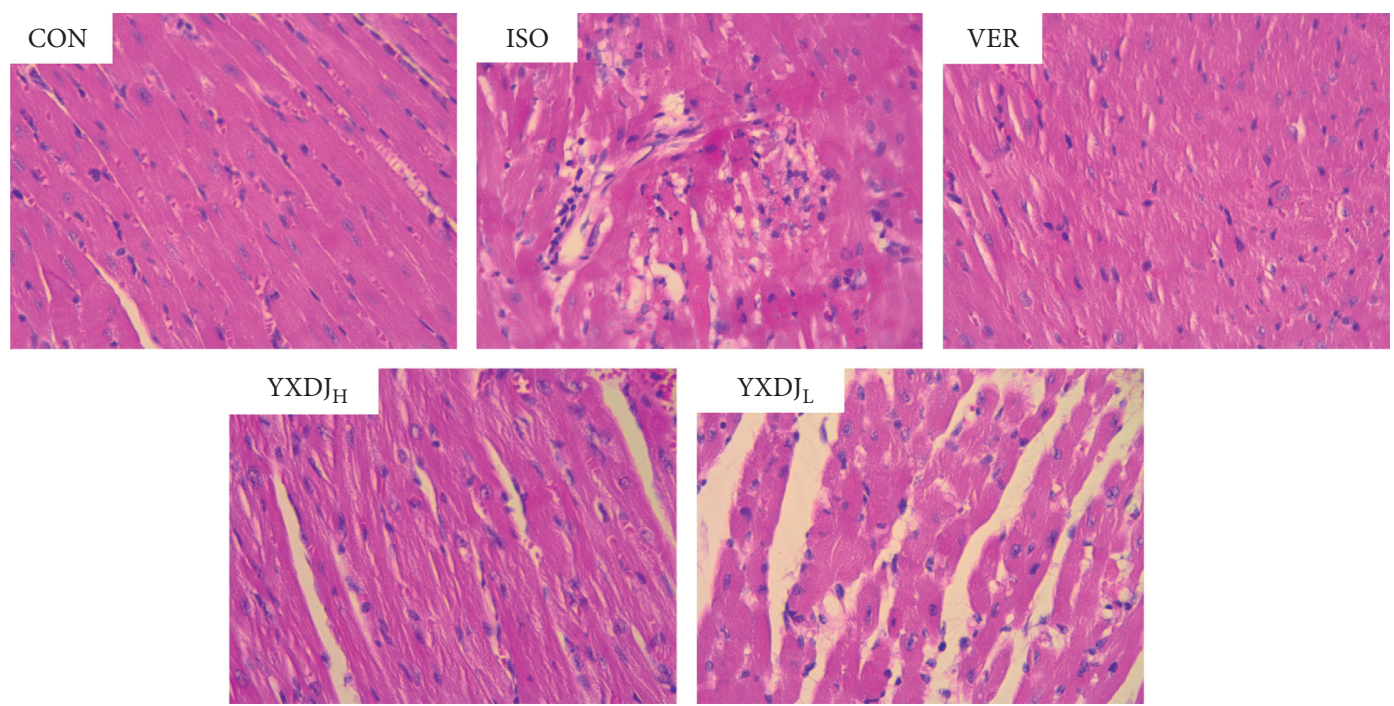

FIGURE 2: Effects of YXDJ on hematoxylin and eosin (H\&E) staining. Representative microscopic photographs of hearts stained with H\&E (magnification: 400x). The myocardial tissues obtained from the CON, ISO, verapamil (VER), and high- and low-dose YXDJ groups. Scale bar: $50 \mu \mathrm{m}$.
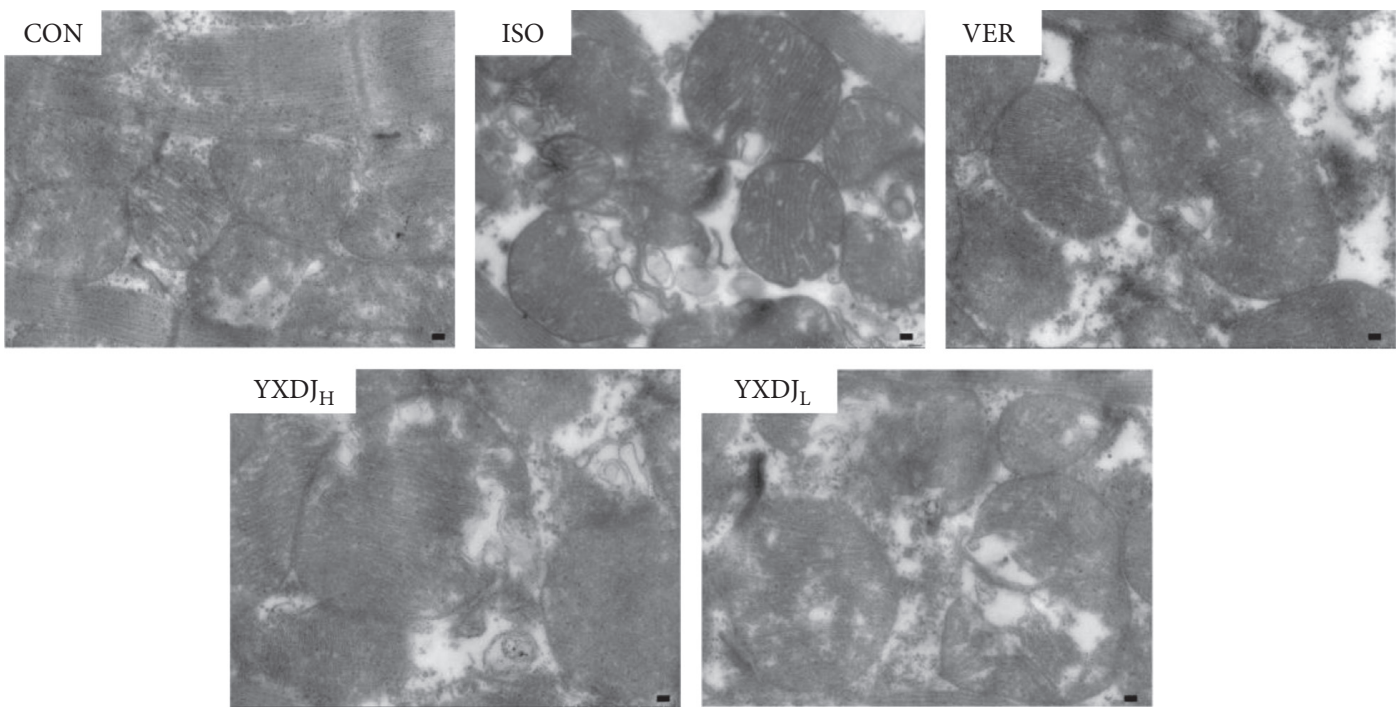

FIGURE 3: Actions of YXDJ on ultrastructural changes. The ultrastructure of cardiac tissues was detected by TEM from the ventricle of the CON, ISO, VER, and high- and low-dose YXDJ groups (magnification: 15000x). Scale bar: $1.0 \mu \mathrm{m}$.

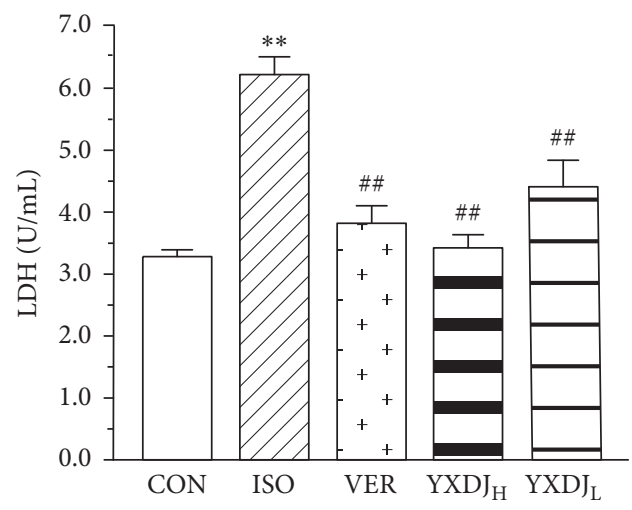

(a)

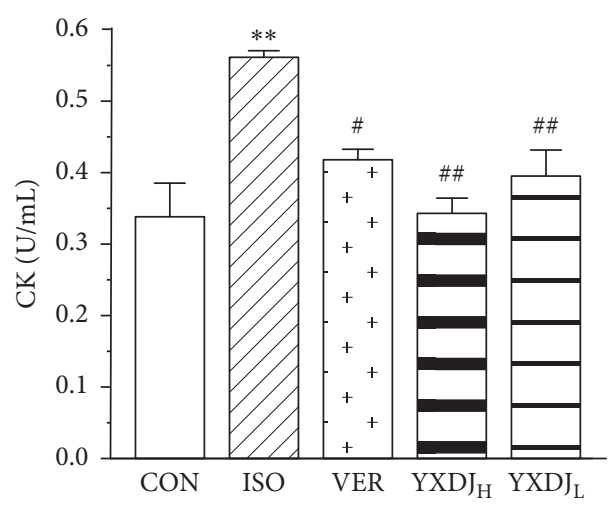

(b)

FIGURE 4: Actions of YXDJ on cardiac marker enzymes. Sera were analyzed for (a) lactate dehydrogenase (LDH) and (b) creatine kinase (CK). Values represent the mean $\pm \mathrm{SE}$, for 10 rats in each group. ${ }^{* *} P<0.05$ versus $\mathrm{CON}$; ${ }^{\#} P<0.05,{ }^{\# \#} P<0.01$ versus ISO. 


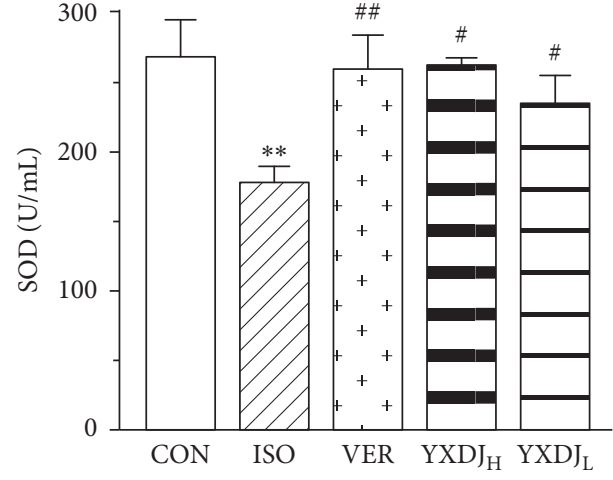

(a)

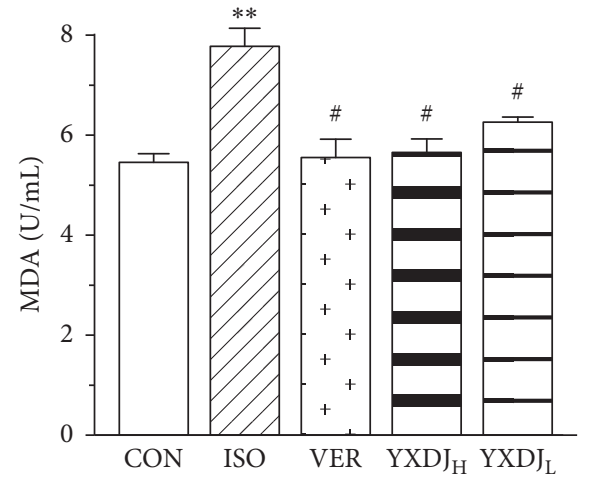

(b)

FIGURE 5: Actions of YXDJ on the superoxide dismutase (SOD) activity (a) and malondialdehyde (MDA) levels (b). Values represent the mean \pm SE, for 10 rats in each group. ${ }^{* *} P<0.05$ versus $C O N$; ${ }^{\#} P<0.05,{ }^{\# \#} P<0.01$ versus ISO.
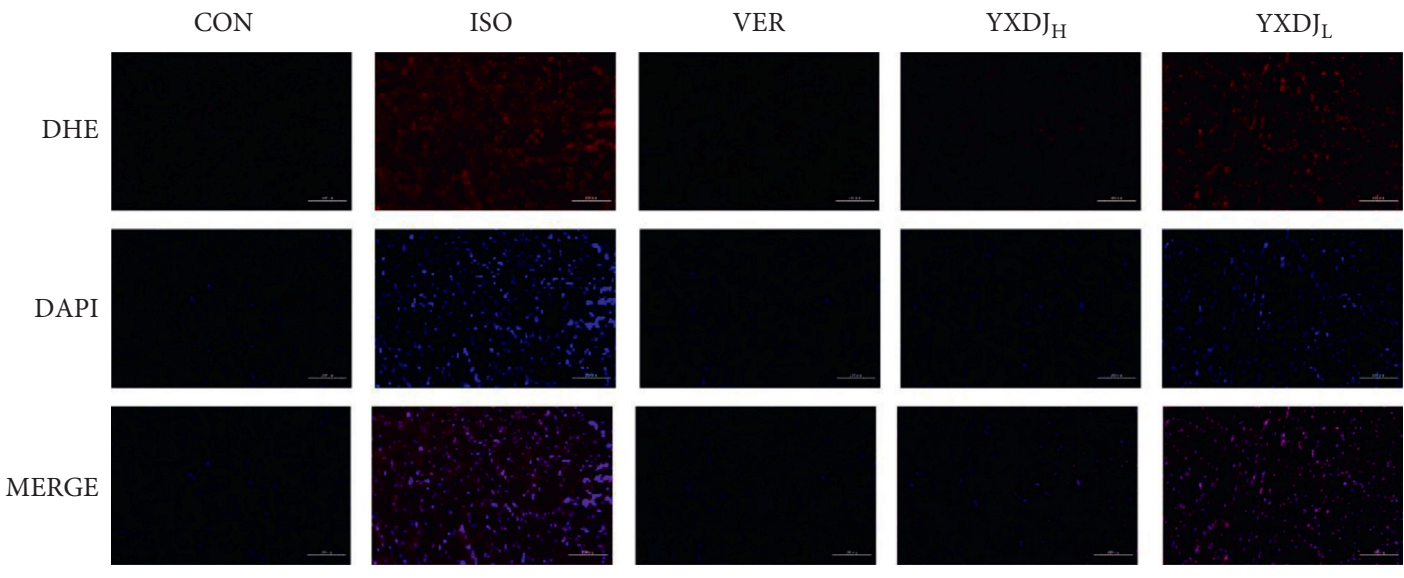

(a)

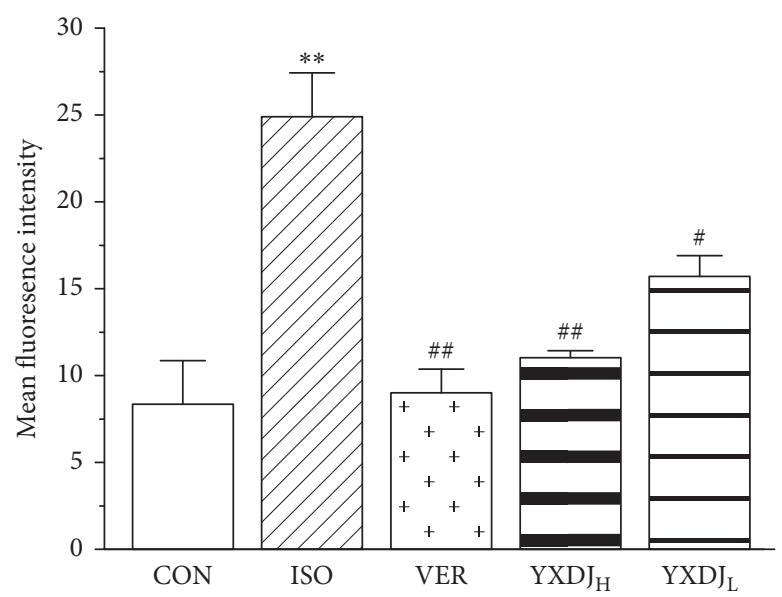

(b)

FIGURE 6: Actions of YXDJ on production of reactive oxygen species (ROS). Representative sections from the cardiac of the CON, ISO, VER, and high- and low-dose YXDJ groups. ROS generation was monitored by dihydroethidium staining. Values represent the mean \pm SE. ${ }^{* *} P<0.05$ versus CON; ${ }^{\#} P<0.05,{ }^{\# \#} P<0.01$ versus ISO. Scale bar: $100 \mu \mathrm{m}$. 


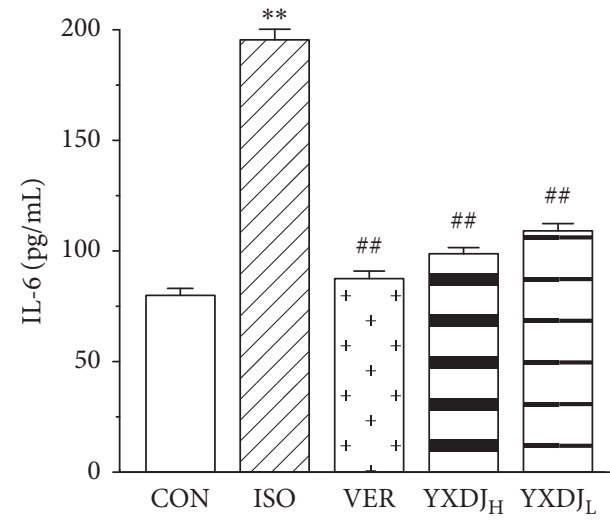

(a)

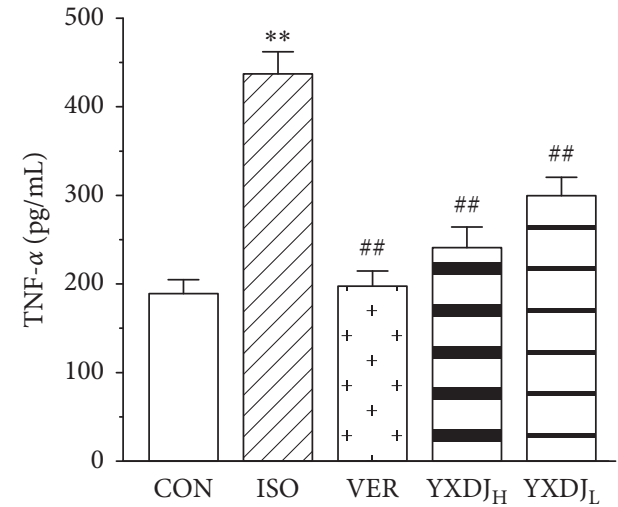

(b)

FIGURE 7: Effects of YXDJ on the expression of interleukin (IL)-6 and tumor necrosis factor (TNF)- $\alpha$ in ISO-induced myocardial infarction (MI): (a) the expression of IL-6 in serum; (b) the expression of TNF- $\alpha$ in serum. Values represent the mean \pm SE, for 10 rats in each group. ${ }^{* *} P<0.05$ versus $\mathrm{CON}$; ${ }^{\#} P<0.05,{ }^{\# \#} P<0.01$ versus ISO.

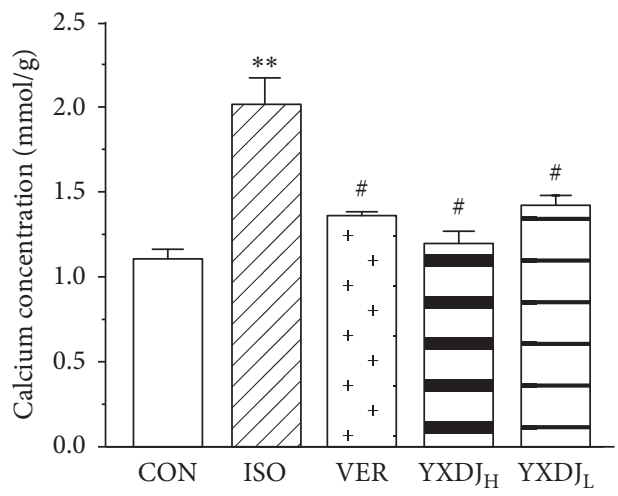

FIGURE 8: Actions of YXDJ on the $\mathrm{Ca}^{2+}$ concentrations. Values represent the mean $\pm \mathrm{SE}$, for 10 rats in each group. ${ }^{* *} P<0.05$ versus CON; ${ }^{\#} P<0.05,{ }^{\#} P<0.01$ versus ISO.

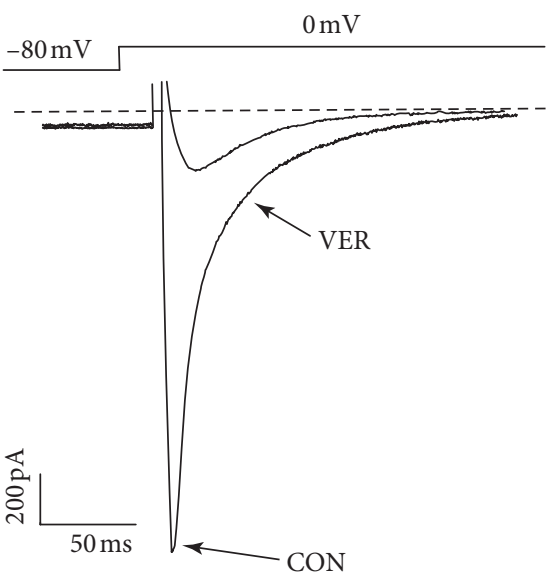

(a)

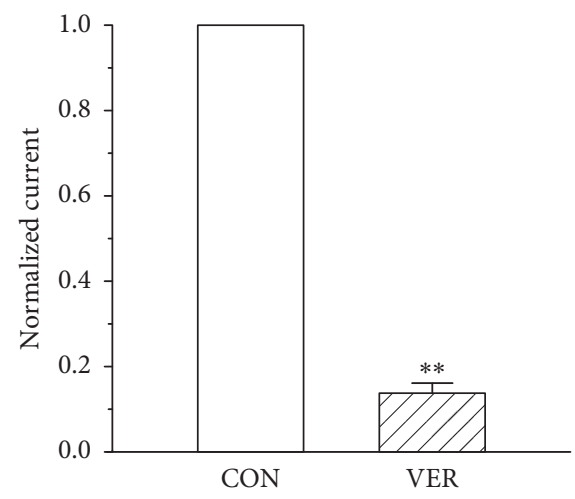

(b)

FIGURE 9: Confirmation of $\mathrm{I}_{\mathrm{Ca}-\mathrm{L}}$ in myocardial cells: (a) representative trace of after treatment by VER (0.1 mM); (b) summary data of (a). Values represent the mean $\pm \mathrm{SE}$, for 7 cells in each group. ${ }^{* *} P<0.05$ versus $\mathrm{CON}$. 


\subsection{Reduction of $I_{C a-L}$ and Cell Shortening by YXDJ}

3.9.1. Confirmation of $I_{C a-L}$. As shown in Figure 9, VER $(0.1 \mathrm{mM})$, as a specific $\mathrm{I}_{\mathrm{Ca}-\mathrm{L}}$ blocker, caused an almost complete abolishment of the currents, indicating that these currents were $\mathrm{Ca}^{2+}$ currents $(P<0.01)$.

3.9.2. Effects of YXDJ on $I_{C a-L}$ of Normal and Ischemic Myocyte Cell. As Figures 10(a)-10(c) show, $\mathrm{I}_{\mathrm{Ca}-\mathrm{L}}$ decreased after treatment with $500 \mu \mathrm{g} / \mathrm{mL}$ YXDJ with an inhibition rate of $52.0 \%$ in normal ventricular myocytes $(P<0.05)$. However, the $\mathrm{I}_{\mathrm{Ca}-\mathrm{L}}$ was almost impossible to recover after washing out with the external solution. As shown in Figures $10(\mathrm{~d})-10(\mathrm{f})$, after the $500 \mu \mathrm{g} / \mathrm{mL}$ YXDJ treatment, $\mathrm{I}_{\mathrm{Ca}-\mathrm{L}}$ was significantly reduced, and the inhibition rate was $47.21 \%$ in ischemic ventricular myocytes $(P<0.05)$; however, the $\mathrm{I}_{\mathrm{Ca}-\mathrm{L}}$ was almost impossible to recover, after washing out with the external solution.

3.9.3. Dose Dependence of YXDJ on $I_{C a-L}$. The time course of the peak ICa-L was progressively decreased by increasing doses of YXDJ $(100,200,300,400$, and $500 \mu \mathrm{g} / \mathrm{mL})$ or VER. As shown in Figure 11, different concentrations of YXDJ were used in the current traces induced by the test potential from -80 to $0 \mathrm{mV}$. The inhibition rates of YXDJ at $100-500 \mu \mathrm{g} / \mathrm{mL} \quad$ (in $100 \mu \mathrm{g} / \mathrm{mL}$ increments) were $7.25 \% \pm 0.94 \%, \quad 18.57 \% \pm 0.89 \%, \quad 32.25 \% \pm 0.47 \%, \quad 44.63 \%$ $\pm 1.69 \%$, and $48.75 \% \pm 1.11 \%$, respectively.

3.9.4. Effects of YXDJ on I-V Relationship. As shown in Figure 12, the effects of YXDJ (300 and $500 \mu \mathrm{g} / \mathrm{mL})$ and $0.1 \mathrm{mM}$ VER on the $\mathrm{I}-\mathrm{V}$ relationship of the $\mathrm{I}_{\mathrm{Ca}-\mathrm{L}}$ are depicted. The current was generated from -60 to $60 \mathrm{mV}$. After the application of YXDJ, I-V curves shifted upward, indicating that YXDJ has a concentration-/time-dependent effect on inhibition of $\mathrm{I}_{\mathrm{Ca}-\mathrm{L}}$. However, the peak potential of $\mathrm{I}_{\mathrm{Ca}-\mathrm{L}}$ and activated potential were markedly unchanged over the measured time period.

3.9.5. Effects of YXDJ on Steady-State Activation and Inactivation of $I_{C a-L}$. As shown in Figure 13, different concentrations of YXDJ (300 and $500 \mu \mathrm{g} / \mathrm{mL}$ ) affected steady-state activation and inactivation of $\mathrm{I}_{\mathrm{Ca}-\mathrm{L}}$. YXDJ at 300 and $500 \mu \mathrm{g} /$ $\mathrm{mL}$ did not change the inactivation and activation of $\mathrm{I}_{\mathrm{Ca}-\mathrm{L}}$. The $V_{1 / 2}$ value for activation of 0,300 , and $500 \mu \mathrm{g} / \mathrm{mL}$ YXDJ was $-2.24 \pm 0.87 \mathrm{mV} / 7.30 \pm 0.73, \quad-1.95 \pm 0.77 \mathrm{mV} / 8.01 \pm$ 0.63 , and $-1.82 \pm 0.98 \mathrm{mV} / 8.25 \pm 0.79$, respectively. The $V_{1 / 2}$ value for inactivation caused by 0,300 , and $500 \mu \mathrm{g} / \mathrm{mL}$ YXDJ was $-26.10506 \pm 0.28256 \mathrm{mV} / 4.76285 \pm 0.23397$, $-26.94475 \pm 0.41879 \mathrm{mV} / 4.96567 \pm 0.36614$, and -30.2175 $2 \pm 0.12523 \mathrm{mV} / 5.77407 \pm 0.1198$, respectively. These data suggest that YXDJ did not alter the activation and inactivation of cardiac $\mathrm{Ca}^{2+}$ gating properties $(P>0.05)$. There were no significant differences between values of $V_{1 / 2}$ in the presence and absence of YXDJ for the normalized inactivation and activation.
3.9.6. Effects of YXDJ on Myocyte Shortening. The representative cell shortening recordings before and after administration of YXDJ are shown in Figure 14. The results indicate that $500 \mu \mathrm{g} / \mathrm{mL}$ YXDJ caused a significant inhibition of cell shortening by $44.4 \% \pm 3.89 \%$.

\section{Discussion}

The goal for this study was to determine whether the YangXinDingJi capsule (YXDJ) was efficacious in protecting against MI by regulating calcium homeostasis. MI causes cardiac tissue damage via reduced levels of nutrients and oxygen due to a temporary decrease or shortage of blood supply. The cardiac muscle disease has partially been reported, and some authors proved an alteration of calcium homeostasis, especially in L-type voltage-gated calcium channel (VGCC) expression and regulation. TCM as therapy for MI has better prospects because of its components and targets [28].

In China, TCM has been considered an alternative and adjuvant approach for preventing cardiovascular disease (CVD) and ischemia injury [29, 30]. While, Zhigancao decoction is a Chinese herbal prescription generally used to treat arrhythmia and palpitation in clinics. YXDJ is a Chinese patent medicine, which was derived from Zhigancao decoction. It is used to treat heart disease in clinics. Although the capsule has been shown to be clinically effective, its protective mechanism on the heart is still unclear. In this paper, we investigated the regulation effect of calcium homeostasis and possible mechanism of YXDJ on ISOinduced MI. However, clinical studies are limited because of the difficulty in obtaining human myocardial samples from myocardial ischemia consumers. In experimental studies, myocardial ischemia models were replicated in animals comparable to what occurs in the human model.

Measurement of cardiac markers in blood is the mainstay for diagnosis of acute myocardial infarction [31]. Therefore, $\mathrm{CK}$ and $\mathrm{LDH}$ were used as biochemical indicators for detecting changes in ISO-induced MI. As shown in Figure 4, CK and $\mathrm{LDH}$ activities decreased after treatment with YXDJ. Meanwhile, as Figure 2 shows, ISO-induced histopathological changes in animal hearts showed that cardiomyocytes appeared smaller, cardiac muscle fibers were disorganized, and vestigial infiltrating inflammatory cells were present. After treatment with YXDJ and VER, histopathological sections show similarly normal structures, slight edema, a small number of inflammatory cardiomyocytes, and clear transverse fringes. The ultrastructure of the myocardium was also changed as shown in Figure 3. Myocardial mitochondria from the ISO group suffered substantial structural damage, and some mitochondria were fused, swollen, or cristae. However, the morphological changes in the YXDJ and VER groups showed significant improvements. In this study, the results of histopathology and cardiac marker enzymes showed that YXDJ caused a significant decreased in ISO-induced myocardial ischemic injury.

Isoprenaline (ISO) is a nonselective $\beta$-adrenoceptor agonist, and it is widely accepted that ISO injection can 


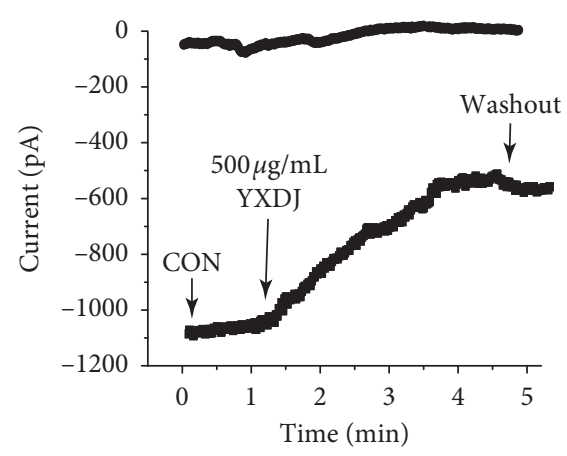

(a)

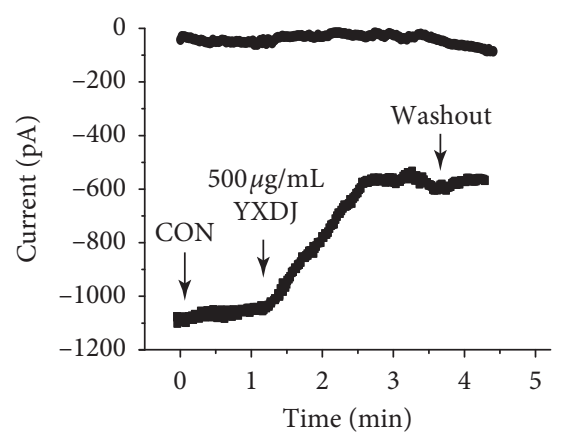

(d)

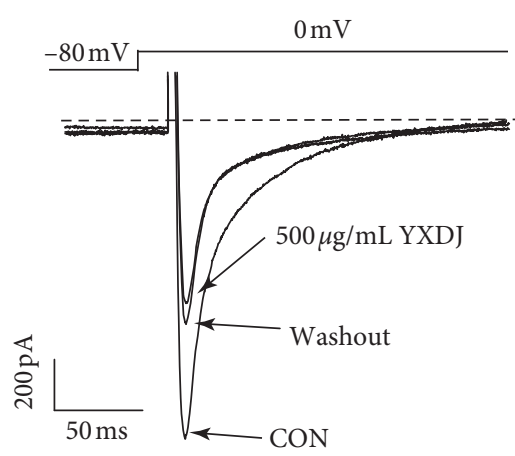

(b)

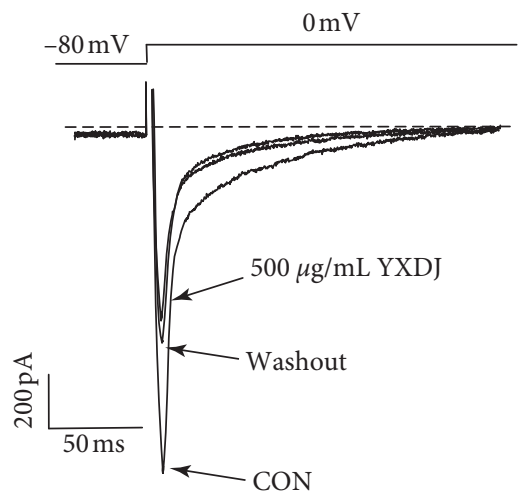

(e)

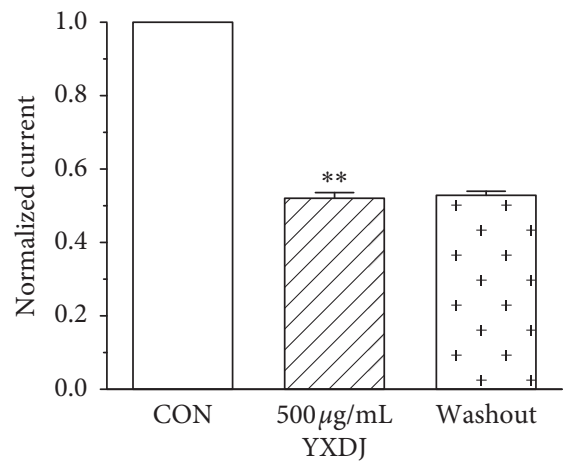

(c)

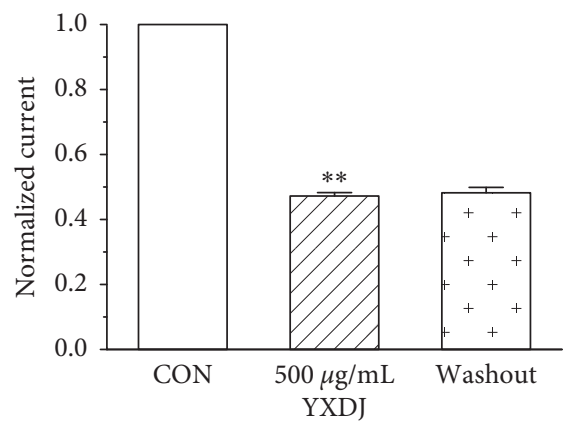

(f)

Figure 10: Actions of YXDJ capsule on $\mathrm{I}_{\mathrm{Ca}-\mathrm{L}}$ of healthy and ischemic cardiomyocytes. Actions of YXDJ capsule on $\mathrm{I}_{\text {Ca-L }}$ of healthy myocardial cells $\left(\mathrm{a}-\mathrm{c}\right.$ ) and ischemic myocardial cells (d-f). YXDJ capsule $\mathrm{I}_{\mathrm{Ca}-\mathrm{L}}$ under control conditions, $500 \mu \mathrm{g} / \mathrm{mL} \mathrm{YXDJ}$, and washout. Values represent the mean \pm SE for 7 cells in each group. ${ }^{* *} P<0.05$ versus CON.

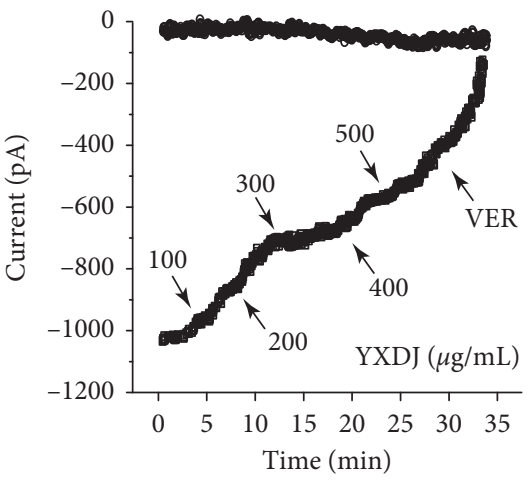

(a)

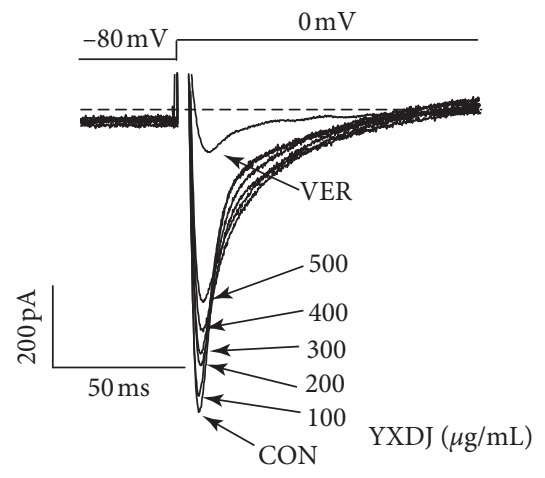

(b)

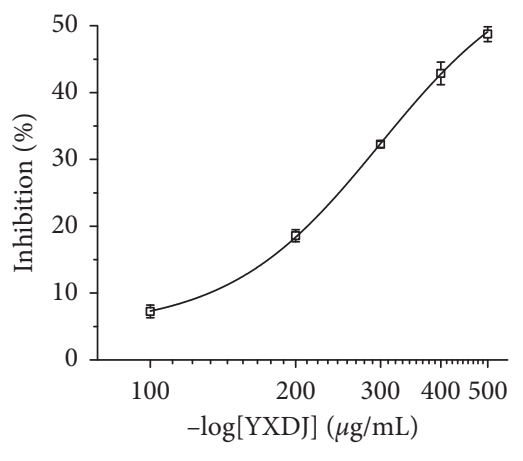

(c)

FIgUre 11: Actions of YXDJ capsule at different concentrations on $\mathrm{I}_{\mathrm{Ca}-\mathrm{L}}$ : (a) time course of exposure to $100-500 \mu \mathrm{g} / \mathrm{mL}$ (in $100 \mu \mathrm{g} / \mathrm{mL}$ increments) and 0.1 mM VER; (b) example of current traces of $\mathrm{I}_{\mathrm{Ca}-\mathrm{L}}$ recorded during exposure to $100-500 \mu \mathrm{g} / \mathrm{mL}$ and $0.1 \mathrm{mM} \mathrm{VER}$; (c) concentration-response curve representing the percent inhibitory of YXDJ. Values represent the mean \pm SE, for 7 cells in each group. ${ }^{* *} P<0.05$ versus CON.

readily induce $\mathrm{MI}$ in animals $[30,32]$. Stimulated $\beta$-adrenergic receptors may induce intracellular $\mathrm{Ca}^{2+}$ overload, inflammation, and accumulation of ROS leading to cardiac problems. In addition, the overloading of $\mathrm{Ca}^{2+}$ can induce arrhythmias and myocardial ischemia $[33,34]$. Thus, blocking the $\mathrm{Ca}^{2+}$ channels and reducing the $\mathrm{Ca}^{2+}$ overload will benefit the treatment of arrhythmias and myocardial ischemic. Our study demonstrates that ISO induction caused MI and arrhythmias, which causes changes in ECGs, such as tachycardia and J-point elevation, while treatment with 

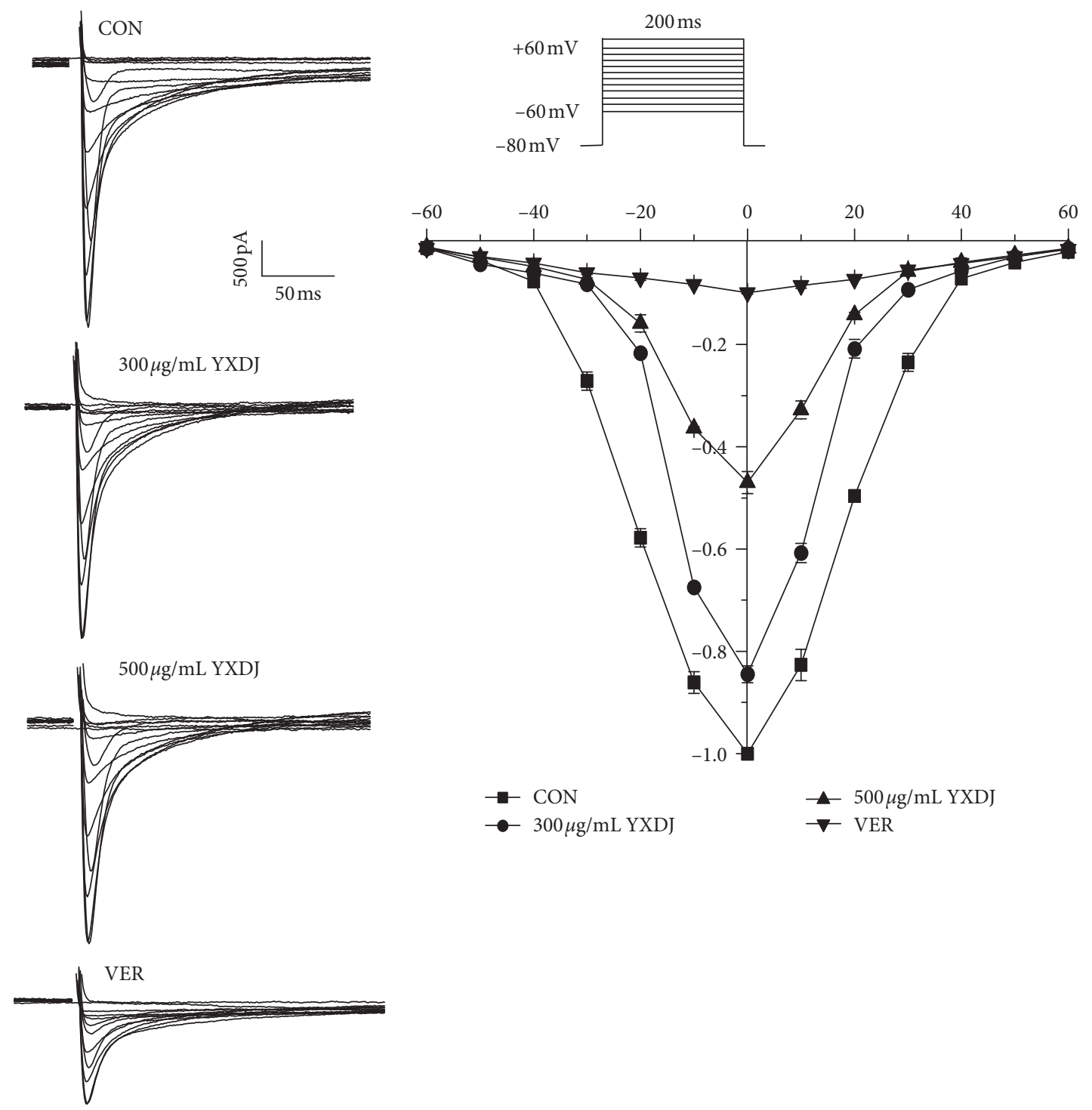

(a)

(b)

Figure 12: Actions of YXDJ capsule on the I-V relationship: (a) representative $\mathrm{I}_{\mathrm{Ca}-\mathrm{L}}$ current recordings and (b) the I-V relationship for $\mathrm{I}_{\mathrm{Ca}-\mathrm{L}}$ under the treatment of Con $(\square)$, YXDJ at $300 \mu \mathrm{g} / \mathrm{mL}(\mathrm{O})$, YXDJ at $500 \mu \mathrm{g} / \mathrm{mL}(\triangle)$, and VER at $0.1 \mathrm{mM}(\nabla)$. Values represent the mean \pm SE for 7 cells in each group.

YXDJ could alleviate tachycardia and reduce J-point elevation. Therefore, YXDJ treatment can reduce the incidence of cardiac arrhythmias and $\mathrm{MI}$ in rats.

MI induced by ISO is mediated by vasospasticity, combined with increased oxygen demand due to its positive inotropic effect, and is related to the oxidative stress. Antioxidant activity is one of the key mechanisms of anti-MI efficacy. There are many kinds of free radicals, and ROS is closely associated with oxidative stress [35]. At the same time, some data have shown that $\mathrm{Ca}^{2+}$ activates the ROSscavenging enzymes, such as SOD and MDA [36]. As shown in Figures 5 and 6, SOD activities, MDA concentrations, and intracellular ROS were detected. The oxidative stress injury in the ISO-induced group was more serious than other groups. After YXDJ treatment, the production of ROS in cardiomyocytes and MDA concentration were obviously reduced, and the activity of SOD was obviously increased. These results indicate that YXDJ can alleviate the oxidative stress response by causing improvements in SOD activity and MDA levels. Therefore, the mechanism of YXDJ in alleviating myocardial ischemic might be related to inhibition of oxidative stress-related reactions. The anti-MI properties of YXDJ were then assessed by studying different inflammatory markers. As shown in Figure 7, evaluation of inflammatory markers showed that ISO induced an obvious increase in TNF- $\alpha$ and IL- 6 levels. Pretreatment with YXDJ and VER led to a decreased in the TNF- $\alpha$ and IL- 6 levels, suggesting that the anti-inflammatory properties were associated with its cardioprotective effects of YXDJ.

$\mathrm{Ca}^{2+}$ is thought to play a significant role in controlling the prooxidant-antioxidant balance. An increase of cytosolic $\mathrm{Ca}^{2+}$ concentration is due to $\mathrm{ROS}$, and $\mathrm{Ca}^{2+}$ increase is a 


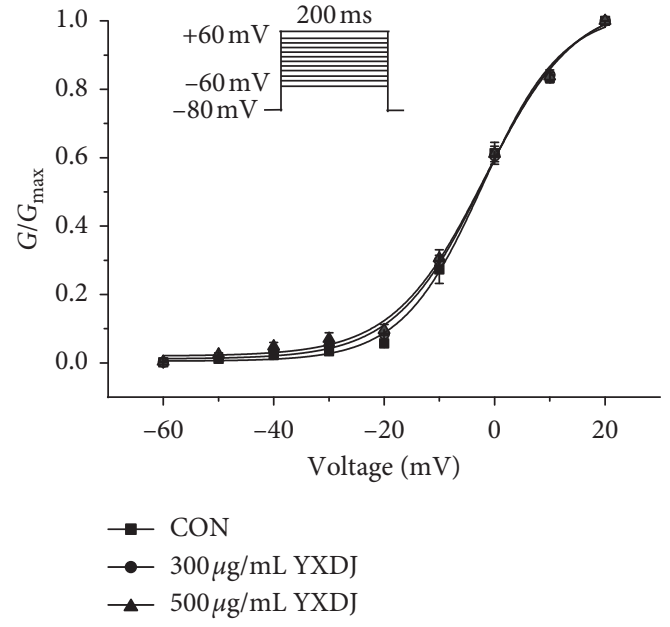

(a)

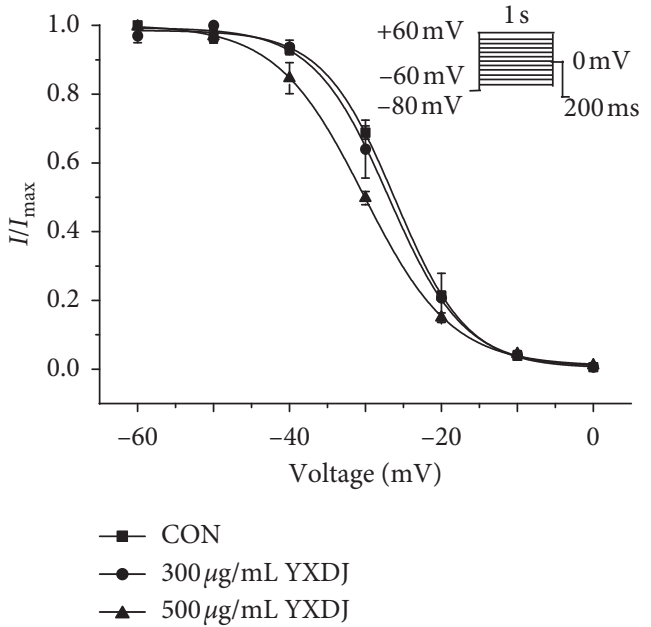

(b)

Figure 13: Actions of YXDJ capsule on steady-state activation and inactivation of $\mathrm{I}_{\mathrm{Ca}-\mathrm{L}}$ : (a) steady-state activation curves and (b) steadystate inactivation of $\mathrm{I}_{\mathrm{Ca}-\mathrm{L}}$ are shown under the treatment of CON $(\square)$, YXDJ at $300 \mu \mathrm{g} / \mathrm{mL}(\mathrm{O})$, and YXDJ at $500 \mu \mathrm{g} / \mathrm{mL}(\triangle)$. Values represent the mean \pm SE for 7 cells in each group.

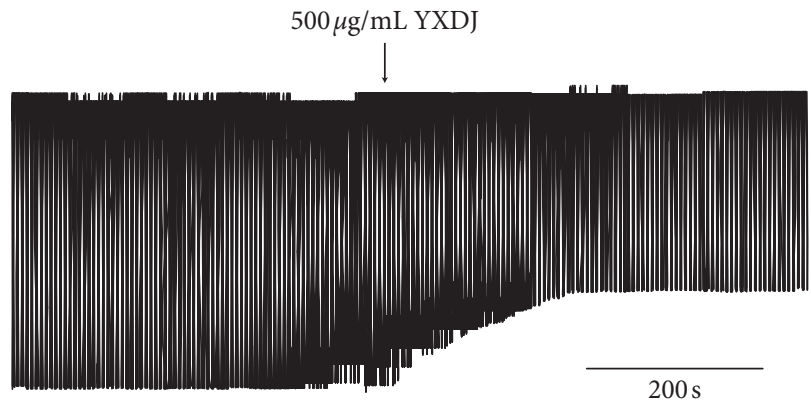

(a)

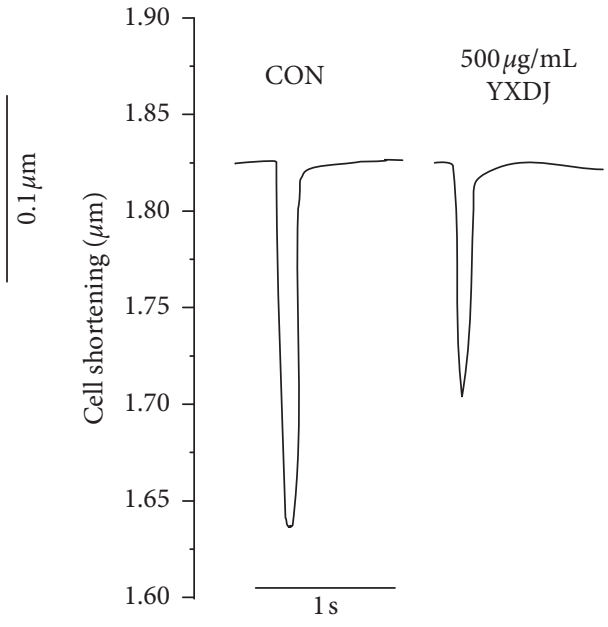

(b)

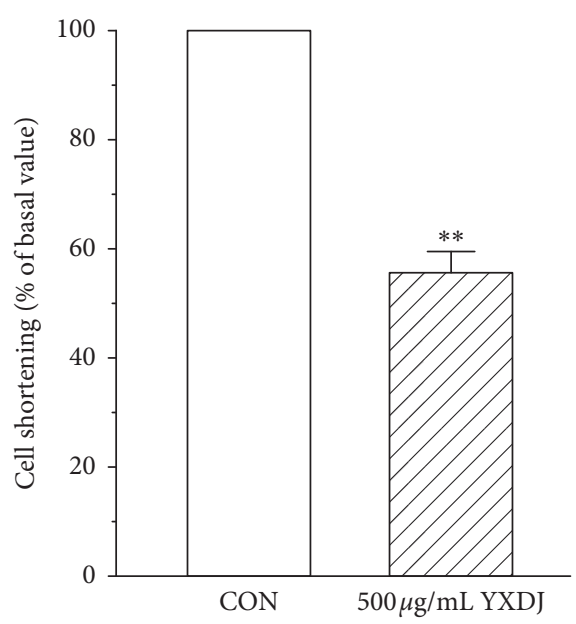

(c)

FIGURE 14: Actions of YXDJ capsule on cell shortening: (a) time parameters of cell shortening recordings; (b) exemplary traces of cell shortening under the CON and with $500 \mu \mathrm{g} / \mathrm{mL}$ YXDJ; (c) summary results of (b). Values represent the mean \pm SE for 7 cells in each group. ${ }^{* *} P<0.05$ versus CON. 

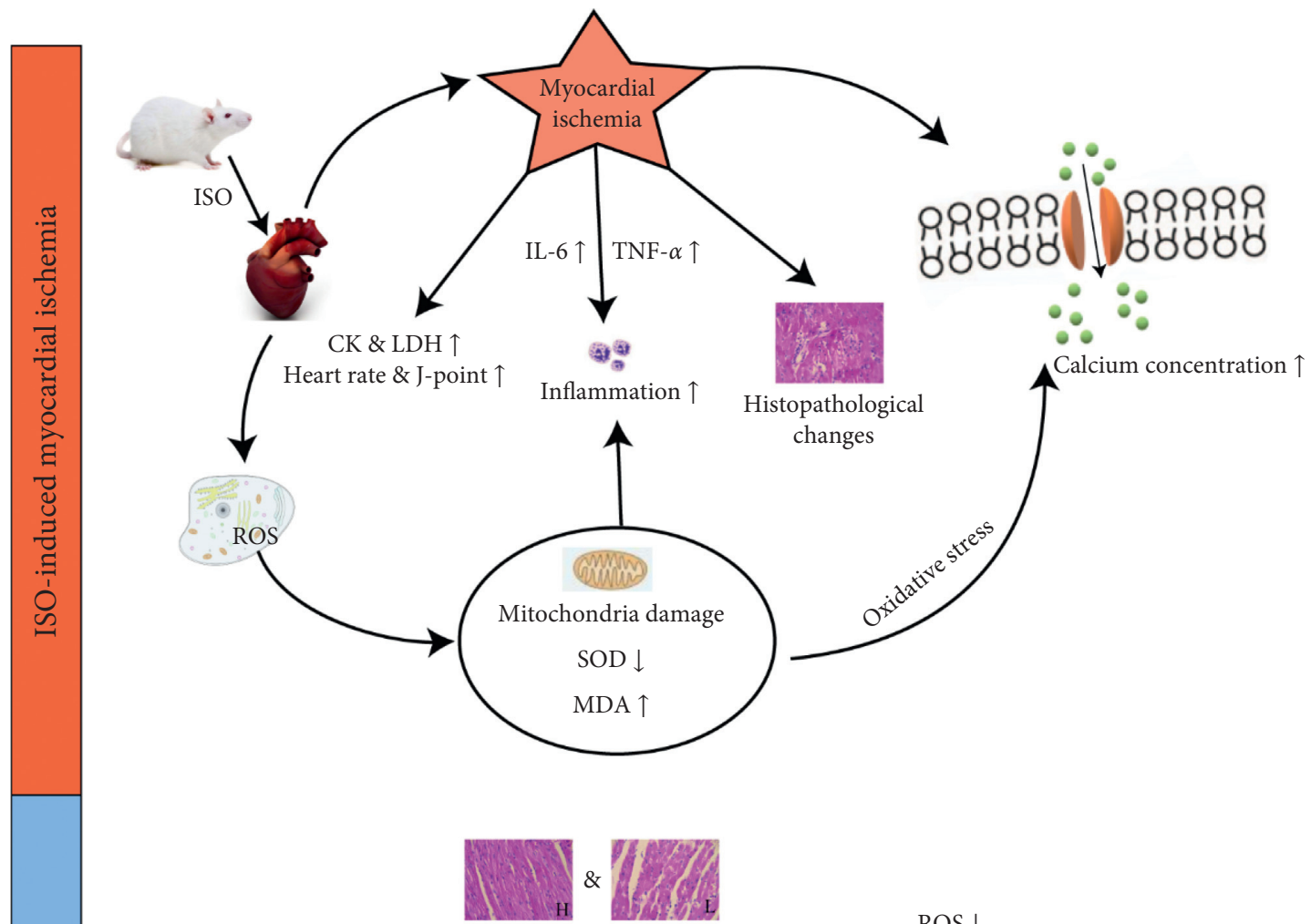

Histopathological

CK \& LDH $\downarrow$

improvement

ROS $\downarrow$

Heart rate \& J-point

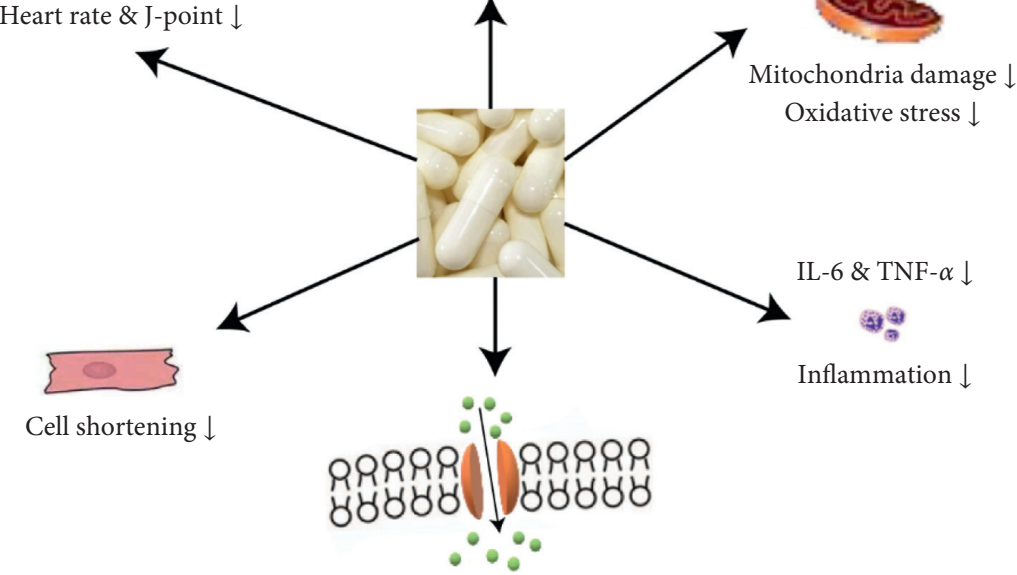

Calcium concentration $\downarrow$

Figure 15: Cardioprotective effects of YXDJ capsule on ISO-induced MI.

constant feature of pathological states associated with oxidative stress [37]. We tested the effects of YXDJ on heart tissues and found that it caused a reduction in the calcium concentration. Therefore, the effects of YXDJ on $\mathrm{Ca}^{2+}$ channels and intracellular conditions were investigated using the whole-cell patch-clamp technique.

The results show that YXDJ led to a reduction in the $\mathrm{I}_{\mathrm{Ca}-\mathrm{L}}$. As shown in Figure 11, YXDJ caused a reduction in $\mathrm{I}_{\mathrm{Ca}-\mathrm{L}}$ in a concentration-dependent manner with an $\mathrm{IC}_{50}$ of $299.26 \mu \mathrm{g} /$ $\mathrm{mL}$. Furthermore, $\mathrm{I}_{\mathrm{Ca}-\mathrm{L}}$ was also reduced by YXDJ at $500 \mu \mathrm{g} /$
$\mathrm{mL}$ in both ischemic ventricular and healthy ventricular myocytes. However, the $\mathrm{I}_{\mathrm{Ca}-\mathrm{L}}$ reverse potential and $\mathrm{I}-\mathrm{V}$ relationship remained unchanged. The heart is an excitable organ, driven by excitation-contraction (EC) coupling that undergoes spontaneous force generation and relaxation cycles. A unique feature of EC coupling is the timely circulation of cytoplasmic calcium under control of a large group of proteins, including $\mathrm{Na}^{+} / \mathrm{Ca}^{2+}$ exchanger (NCX), phospholamban-regulated $\mathrm{Ca}^{2+}$-ATPase (SERCA), and ryanodine receptor (RyR) [38]. The process of $\mathrm{Ca}^{2+}$ induced $\mathrm{Ca}^{2+}$ release 
(CICR) is caused by $\mathrm{Ca}^{2+}$ inflow that triggers $\mathrm{Ca}^{2+}$ release from the sarcoplasmic reticulum (SR). However, CICR provides the necessary signal to initiate mechanical shortening of the cell contraction mechanism and can lead to several-fold increase in cytosolic $\mathrm{Ca}^{2+}$ concentration [39]. Nevertheless, the mechanisms for altering calcium homeostasis have still not been clearly established. The function of calcium channel antagonists is to reduce the entering rate of $\mathrm{Ca}^{2+}$ and turn off the calcium channel. Calcium antagonists are introduced into the therapeutic armory because of their specific effects on oxygen consumption, myocardial contractility and myocardial blood supply. In this present study, patch-clamp methods were used to examine excitationcontraction (EC) coupling, and we found that YXDJ had a significant inhibitory effect on $\mathrm{I}_{\mathrm{Ca}-\mathrm{L}}$. It seems that YXDJ could be used as a cardioprotective agent to inhibit $\mathrm{I}_{\mathrm{Ca}-\mathrm{L}}$ and reduce myocardial contractility. These results suggest that YXDJ caused the $\mathrm{I}_{\mathrm{Ca}-\mathrm{L}}$ inhibition primarily by reducing the $\mathrm{Ca}^{2+}$ current amplitude.

In addition, contractility was inhibited by YXDJ. This result can be explained by the process of myocardial contraction. Contraction is related not only to intracellular $\mathrm{Ca}^{2+}$ concentration but also to intracellular proteins that are involved in contraction. We utilized an IonOptix MyoCam detection system to examine the impact of YXDJ on rat ventricular myocytes. In this study, YXDJ significantly reduced the $\mathrm{Ca}^{2+}$ contractility, which may be related to the inhibition of $\mathrm{I}_{\mathrm{Ca}-\mathrm{L}}$. Furthermore, the present study focused on the effect of YXDJ in the of MI rats. To investigate the mechanism through which YXDJ exerts antiarrhythmic effect, it is necessary to study the related ion channels. However, the L-type $\mathrm{Ca}^{2+}$ channel is involved in the predominant mechanism responsible for the influx of $\mathrm{Ca}^{2+}$ in cardiac cells. We found that YXDJ can inhibit LTCC reducing calcium ions released, thereby reducing free intracellular $\mathrm{Ca}^{2+}$ and inhibiting cell shortening. This series of studies reveal that YXDJ can effectively reduce calcium overload and effectively relieve arrhythmias and myocardial ischemia.

Our data suggest that YXDJ could inhibit the increase in $\mathrm{Ca}^{2+}$ concentration via a decrease in the extracellular $\mathrm{Ca}^{2+}$ influx. Sufficient evidence links YXDJ to cardioprotection via inhibition of $\mathrm{I}_{\mathrm{Ca}-\mathrm{L}}$ and myocardial contractility.

\section{Conclusion}

The results demonstrated the cardioprotective effects of YXDJ in ISO-induced MI (see Figure 15). The mechanism may be related to regulating oxidative stress, anti-inflammatory effects, and reduction of calcium inflow via LTCC inhibition. These results provide the basis for further understanding the molecular mechanism of the beneficial effects of YXDJ on IHD.
Abbreviations
CK: $\quad$ Creatine kinase
CVD: Cardiovascular disease
DHE: Dihydroethidium

ECG: Electrocardiogram

H\&E: Hematoxylin and eosin

$\mathrm{IC}_{50}$ : Concentration required to inhibit transport by $50 \%$

$\mathrm{I}_{\mathrm{Ca}-\mathrm{L}}$ : L-type calcium current

IHD: Ischemic heart disease

IL-6: Interleukin-6

LTCC: L-type $\mathrm{Ca}^{2+}$ channel

ISO: Isoproterenol

LDH: Lactate dehydrogenase

MDA: Malondialdehyde

MI: $\quad$ Myocardial ischemia

NCX: $\quad \mathrm{Na}^{+} / \mathrm{Ca}^{2+}$ exchanger

ROS: Reactive oxygen species

RyR: Ryanodine receptor

SOD: Superoxide dismutase

TNF- $\alpha$ : Tumor necrosis factor- $\alpha$

TCM: Traditional Chinese medicine

TEM: Transmission electron microscope

YXDJ: YangXinDingJi capsule.

\section{Data Availability}

The data used to support the findings of this study are available from the corresponding author upon request.

\section{Ethical Approval}

This study was approved by the Ethics Committee for Animal Experiments of Hebei University of Chinese Medicine (approved number: DWLL2020004 and date: Jan. 9, 2020).

\section{Conflicts of Interest}

The authors declare that there are no conflicts of interest.

\section{Authors' Contributions}

Li Chu, Xue Han, and Ziliang Li designed the research; Miaomiao Liu, Xue Han, and Yurun Xue performed the experiments; Miaomiao Liu, Yingran Liang, Yucong Xue, and Yurun Xue analyzed the data; Miaomiao Liu, Li Chu, and Ziliang Li wrote the manuscript; and Li Chu revised the manuscript.

\section{Acknowledgments}

This work was supported by the Research Foundation of Hebei University of Chinese Medicine (KTZ2019043 and YTZ2019003).

\section{References}

[1] H. Thomas, J. Diamond, A. Vieco et al., "Global atlas of cardiovascular disease 2000-2016: the path to prevention and control," Global Heart, vol. 13, no. 3, pp. 143-163, 2018.

[2] K. Chaudhuri, D. Kaye, J. Headrick et al., "Myocardial ischemia-reperfusion injury, antioxidant enzyme systems, and selenium: a review," Current Medicinal Chemistry, vol. 14, no. 14, pp. 1539-1549, 2007. 
[3] A. Caliskan, C. Yavuz, O. Karahan et al., "Iloprost reduces myocardial edema in a rat model of myocardial ischemia reperfusion," Perfusion, vol. 29, no. 3, pp. 260-264, 2014.

[4] A. P. Waldenström, Å. C. Hjalmarson, and L. Thornell, "A possible role of noradrenaline in the development of myocardial infarction: an experimental study in the isolated rat heart," American Heart Journal, vol. 95, no. 1, pp. 43-51, 1978.

[5] Z. Nichtova, M. Novotova, E. Kralova, and T. Stankovicova, "Morphological and functional characteristics of models of experimental myocardial injury induced by isoproterenol," General Physiology and Biophysics, vol. 31, no. 2, pp. 141-151, 2012.

[6] H. M. Stankovicova and L. C. Hool, "The L-type $\mathrm{Ca}^{2+}$ channel: a mediator of hypertrophic cardiomyopathy," Channels, vol. 11, no. 1, pp. 5-7, 2017.

[7] H. M. Viola, P. G. Arthur, and L. C. Hool, "Evidence for regulation of mitochondrial function by the L-type $\mathrm{Ca}^{2+}$ channel in ventricular myocytes," Journal of Molecular and Cellular Cardiology, vol. 46, no. 6, pp. 1016-1026, 2009.

[8] M. F. Navedo, G. C. Amberg, V. S. Votaw, and L. F. Santana, "Constitutively active L-type $\mathrm{Ca}^{2+}$ channels," Proceedings of the National Academy of Sciences of the United States of America, vol. 102, no. 31, pp. 11112-11117, 2005.

[9] P. Santana, C. E. Van Hove, J. van Langen et al., "Contribution of transient and sustained calcium influx, and sensitization to depolarization-induced contractions of the intact mouse aorta," BMC Physiology, vol. 12, no. 1, p. 9, 2012.

[10] D. M. Bers, "Ca regulation in cardiac muscle," Medicine and Science in Sports and Exercise, vol. 23, no. 10, pp. 1157-1162, 1991.

[11] A. W. Harman and M. J. Maxwell, "An evaluation of the role of calcium in cell injury," Annual Review of Pharmacology and Toxicology, vol. 35, no. 1, pp. 129-144, 1995.

[12] X. Yang, Y. Chen, Y. Li et al., "Effects of Wenxin Keli on cardiac hypertrophy and arrhythmia via regulation of the calcium/calmodulin dependent kinase II signaling pathway," Biomed Research International, vol. 2017, Article ID 1569235, 12 pages, 2017.

[13] S. Abdoul-Azize, C. Buquet, J.-P. Vannier, and I. Dubus, "Pyr3, a TRPC3 channel blocker, potentiates dexamethasone sensitivity and apoptosis in acute lymphoblastic leukemia cells by disturbing $\mathrm{Ca}^{2+}$ signaling, mitochondrial membrane potential changes and reactive oxygen species production," European Journal of Pharmacology, vol. 784, pp. 90-98, 2016.

[14] M. A. Dubus, E. Tseliou, B. Sun et al., "Therapeutic efficacy of cardiosphere-derived cells in a transgenic mouse model of non-ischaemic dilated cardiomyopathy," European Heart Journal, vol. 36, no. 12, pp. 751-762, 2015.

[15] X. Cheng, B. Li, Y. Zhao et al., "Protective effects of antioxidants on chronic intermittent hypoxia-induced cardiac remodeling in mice," Zhonghua Xin Xue Guan Bing Za Zhi, vol. 42, no. 11, pp. 944-950, 2014.

[16] D. P. Jones, "Redefining oxidative stress," Antioxid Redox Signal, vol. 8, no. 9-10, pp. 1865-1879, 2006.

[17] R. Rodrigo, M. Libuy, F. Feliú, and D. Hasson, "Oxidative stress-related biomarkers in essential hypertension and ischemia-reperfusion myocardial damage," Disease Markers, vol. 35, no. 6, pp. 773-790, 2013.

[18] A. Hasson, H. H. Hughie, and P. A. Lucchesi, "Regulation of hypertrophic and apoptotic signaling pathways by reactive oxygen species in cardiac myocytes," Antioxidants \& Redox Signaling, vol. 5, no. 6, pp. 731-740, 2003.

[19] H. Katsumi, M. Nishikawa, H. Yasui, F. Yamashita, and M. Hashida, "Prevention of ischemia/reperfusion injury by hepatic targeting of nitric oxide in mice," Journal of Controlled Release, vol. 140, no. 1, pp. 12-17, 2009.

[20] H.-W. Yamashita, H.-J. Liu, H. Cao, Z.-Y. Qiao, and Y.-W. Xu, "Diosgenin protects rats from myocardial inflammatory injury induced by ischemia-reperfusion," Medical Science Monitor, vol. 24, pp. 246-253, 2018.

[21] G. Qiao, O. Dewald, and N. Frangogiannis, "Inflammatory mechanisms in myocardial infarction," Current Drug Target -Inflammation \& Allergy, vol. 2, no. 3, pp. 242-256, 2003.

[22] P. Hao, F. Jiang, J. Cheng, L. Ma, Y. Zhang, and Y. Zhao, "Traditional Chinese medicine for cardiovascular disease: evidence and potential mechanisms," Journal of the American College of Cardiology, vol. 69, no. 24, pp. 2952-2966, 2017.

[23] W. Ma, X. Xiong, B. Feng, R. Yuan, F. Chu, and H. Liu, "Classic herbal formula Zhigancao decoction for the treatment of premature ventricular contractions (PVCs): a systematic review of randomized controlled trials," Complementary Therapies in Medicine, vol. 23, no. 1, pp. 100-115, 2015.

[24] J. Yuan, Y. Lu, H. Yan et al., "Application of the myocardial tissue/silicon substrate microelectrode array technology on detecting the effection of Zhigancao decoction medicated serum on cardiac electrophysiology," International Journal of Clinical and Experimental Medicine, vol. 8, no. 2, pp. 20172023, 2015.

[25] H.-X. Wang, L.-F. Li, R. An, X.-M. Cheng, C.-H. Wang, and X.-H. Wang, "Simultaneous determination of four constituents in Yangxin Dingji capsules by HPLC," Chinese Traditional Patent Medicine, vol. 39, no. 1, pp. 98-101, 2017.

[26] X. Zhao, "Curative efficacy of Yangxindingji capsule in treatment of coronary heart disease and effects on cardiovascular events," Chinese Journal of Microcirculation, vol. 28, no. 3, pp. 31-34, 2018.

[27] A. E. Belevych, C. Sims, and R. D. Harvey, "ACh-induced rebound stimulation of L-type $\mathrm{Ca}^{2+}$ current in guinea-pig ventricular myocytes, mediated by Gbetagamma-dependent activation of adenylyl cyclase," Journal of Physiology, vol. 536, no. 3, pp. 677-692, 2001.

[28] F. Liu, Z.-Z. Huang, Y.-H. Sun et al., "Four main active ingredients derived from a traditional Chinese medicine Guanxin Shutong capsule cause cardioprotection during myocardial ischemia injury calcium overload suppression," Phytotherapy Research, vol. 31, no. 3, pp. 507-515, 2017.

[29] J.-Y. Li, Q. Li, Z.-Z. Ma, and J.-Y. Fan, "Effects and mechanisms of compound Chinese medicine and major ingredients on microcirculatory dysfunction and organ injury induced by ischemia/reperfusion," Pharmacology \& Therapeutics, vol. 177, pp. 146-173, 2017.

[30] Y. C. Fan, L. Yan, C. S. Pan et al., "The contribution of different components in QiShenYiQi pills(R) to its potential to modulate energy metabolism in protection of ischemic myocardial injury," Frontiers in Physiology, vol. 9, p. 389, 2018.

[31] A. H. B. Wu, M. Panteghini, F. S. Apple, R. H. Christenson, F. Dati, and J. Mair, "Biochemical markers of cardiac damage: from traditional enzymes to cardiac-specific proteins. IFCC subcommittee on standardization of cardiac markers (SSCM)," Scandinavian Journal of Clinical and Laboratory Investigation, vol. 59, no. sup230, pp. 74-82, 1999.

[32] O. E. Panteghini, "Cardiac hypertrophy induced by sustained $\beta$-adrenoreceptor activation: pathophysiological aspects," Heart Failure Reviews, vol. 12, no. 1, pp. 66-86, 2007.

[33] Y. Xing, Y. Gao, J. Chen et al., "Wenxin-Keli regulates the calcium/calmodulin-dependent protein kinase II signal 
transduction pathway and inhibits cardiac arrhythmia in rats with myocardial infarction," Evidence-Based Complementary and Alternative Medicine, vol. 2013, Article ID 464508, 15 pages, 2013.

[34] Y. Chen, Y. Li, L. Guo et al., "Effects of Wenxin Keli on the action potential and L-type calcium current in rats with transverse aortic constriction-induced heart failure," Evidence-Based Complementary and Alternative Medicine, vol. 2013, Article ID 572078, 12 pages, 2013.

[35] H. Inafuku, Y. Kuniyoshi, S. Yamashiro et al., "Determination of oxidative stress and cardiac dysfunction after ischemia/ reperfusion injury in isolated rat hearts," Annals of Thoracic and Cardiovascular Surgery, vol. 19, no. 3, pp. 186-194, 2013.

[36] A. E. D. Arakaki, M. A. Ilian, J. D. Morton, L. Vanhanen, J. R. Sedcole, and R. Bickerstaffe, "Effect of calcium chloride, zinc chloride, and water infusion on metmyoglobin reducing activity and fresh lamb color," Journal of Animal Science, vol. 83, no. 9, pp. 2189-2204, 2005.

[37] L. Vanhanan, S. Amoroso, A. Pannaccione et al., "Apoptosis induced in neuronal cells by oxidative stress: role played by caspases and intracellular calcium ions," Toxicology Letters, vol. 139, no. 2-3, pp. 125-133, 2003.

[38] G. Gong, X. Liu, and W. Wang, "Regulation of metabolism in individual mitochondria during excitation-contraction coupling," Journal of Molecular and Cellular Cardiology, vol. 76, pp. 235-246, 2014.

[39] J. L. Greenstein, R. Hinch, and R. L. Winslow, "Mechanisms of excitation-contraction coupling in an integrative model of the cardiac ventricular myocyte," Biophysical Journal, vol. 90, no. 1, pp. 77-91, 2006. 